\title{
Marshall-Olkin Log-Logistic Extended Weibull Distribution : Theory, Properties and Applications
}

\author{
Lornah Lepetu ${ }^{1}$, Broderick O. Oluyede ${ }^{2}$, Boikanyo Makubate ${ }^{3}$, Susan Foya ${ }^{4}$ and Precious Mdlongwa ${ }^{5}$ \\ ${ }^{1,3,5}$ Botswana International University of Science and Technology, Palapye, Botswana \\ ${ }^{2}$ Georgia Southern University, Statesboro, GA, USA \\ ${ }^{4}$ First National Bank, Goborone, Botswana
}

\begin{abstract}
Marshall and Olkin (1997) introduced a general method for obtaining more flexible distributions by adding a new parameter to an existing one, called the Marshall-Olkin family of distributions. We introduce a new class of distributions called the Marshall - Olkin Log-Logistic Extended Weibull (MOLLEW) family of distributions. Its mathematical and statistical properties including the quantile function hazard rate functions, moments, conditional moments, moment generating function are presented. Mean deviations, Lorenz and Bonferroni curves, Rényi entropy and the distribution of the order statistics are given. The Maximum likelihood estimation technique is used to estimate the model parameters and a special distribution called the Marshall-Olkin Log Logistic Weibull (MOLLW) distribution is studied, and its mathematical and statistical properties explored. Applications and usefulness of the proposed distribution is illustrated by real datasets.
\end{abstract}

Keywords: Marshall-Olkin, Log-Logistic Weibull distribution,

Maximum Likelihood Estimation.

\section{Introduction}

Marshall and Olkin (1997) derived an important method of including an extra shape parameter to a given baseline model thus defining an extended distribution. The Marshall-Olkin transformation provides a wide range of behaviors with respect to the baseline distribution (Santos-Neo et al. 2014). Adding parameters to a well-established distribution is a time-honored device for obtaining more flexible new families of distributions (Cordeiro and Lemonte 2011). Several new models have been proposed that are some way related to the Weibull distribution which is a very popular distribution for modelling data in reliability, engineering and biological studies. Extended forms of the Weibull distribution and applications in the literature include Xie 
et al. (2002), Bebbington et al. (2007), Cordeiro et al. (2010) and Silva et al. (2010). When modeling monotone hazard rates the Weibull distribution may be an initial choice because of its negatively and positively skewed density shapes, it however does not provide a reasonable fit for modeling phenomenon with non-monotone failure rates such as bathtub shaped and unimodal failure rates which are common in reliability, engineering and biological Sciences.

This paper employs the Marshall-Olkin transformation to the Log-Logistic Weibull distribution to obtain a new more flexible distribution to describe reliability data. Marshall and Olkin applied the transformation and generalized the exponential and Weibull distribution. Subsequently, the Marshall- Olkin transformation was applied to several well-known distributions: Weibull (Ghittany et al. 2005, Zhang and Xie 2007). More recently, general results have been addressed by Barreto-Souza et al. (2013) and Cordeiro and Lemonte (2013). Santos-Nero et al. (2014) introduces a new class of models called the Marshall-Olkin extended Weibull family of distributions which defines at least twenty-one special models. Chakraborty and Handique (2017) presented the generalized Marshall-Olkin Kumaraswamy-G distribution. Lazhar (2016) developed and studied the properties of the Marshall-Olkin extended generalized Gompertz distribution. Kumar (2016) discussed the ratio and inverse moments of Marshall-Olkin extened Burr Type III distribution based on lower generalized order statistics. However these authors do not employ the Marshall-Olkin transformation in extending the Log-Logistic Weibull distribution (Oluyede et al. 2016). The combined distribution of Log- Logistic and Weibull is obtained from the product of the reliability or survival functions of the Log-logistic and Weibull distribution. The Marshall-Olkin transformation is then employed to obtain a new model called Marshall-Olkin Log-Logistic Extended Weibull (MOLLEW) distribution. A motivation for developing this model is the advantages presented by this extended distribution with respect to having a hazard function that exhibits increasing, decreasing and bathtub shapes, as well as the versatility and flexibility of the log-logistic and Weibull distributions in modeling lifetime data.

The results in this paper are organized in the following manner. In section 2, we present the extended model called Marshall-Olkin Log-Logistic Extended Weibull (MOLLEW) distribution, quantile function and hazard function. In section 3, moments, moment generating function and conditional moments are presented. Lorenz and Bonferroni curves are also presented in section 3. Section 4 contain results on Rényi entropy. In Section 5, maximum likelihood estimates of the model parameters are given. In section 6 , the special case, that is, the new model called the Marshall-Olkin Log-Logistic Weibull (MOLLW) distribution its sub models, density expansion, quantile function, hazard and reverse hazard function, moments, moment generating function, conditional moments, Lorenz and Bonferroni curves and Rényi entropy are derived. Max- 
imum likelihood estimates of the model parameters are given in section 7 . A Monte Carlo simulation study to examine the bias and mean square error of the maximum likelihood estimates are presented in section 8. Section 9 contains applications of the new model to real data sets. A short conclusion remark is given in section 10 .

\section{Marshall-Olkin Log-Logistic Extended Weibull Distribution}

In this section, the model, hazard function and quantile function of the MarshallOlkin Log-Logistic Extended Weibull (MOLLEW) distribution are presented. First, we present the class of extended Weibull distributions, Log-Logistic Weibull distribution and the Marshall-Olkin Log-Logistic Extended Weibull distribution.

Gurvich et al. (1997) pioneered the class of extended Weibull (EW) distributions. The cumulative distribution function (cdf) is given by

$$
G(x ; \alpha, \xi)=1-\exp [-\alpha H(x ; \xi)], \quad x \in \mathcal{D}, \alpha>0,
$$

where $\mathcal{D}$ is a subset of $R$, and $H(x ; \xi)$ is a non-negative function that depends on the vector of parameters $\xi$. The corresponding probability density function (pdf) is given by

$$
g(x ; \alpha, \xi)=\alpha \exp [-\alpha H(x ; \xi)] h(x ; \xi)
$$

where $h(x ; \xi)$ is the derivative of $H(x ; \xi)$. The choice of the function $H(x ; \xi)$ leads to different models including for example, exponential distribution with $H(x ; \xi)=x$, Rayleigh distribution is obtained from $H(x ; \xi)=x^{2}$ and Pareto distribution from setting $H(x ; \xi)=\log (x / k)$. Now consider a general case called log-logistic extended Weibull family of distributions. The distribution is obtained via the use of competing risk model and is given by combining both the log-logistic and extended Weibull family of distributions as given below (Oluyede et al. 2016). The corresponding pdf is given by

$$
f(x ; c, \alpha, \xi)=e^{-\alpha H(x ; \xi)}\left(1+x^{c}\right)^{-1}\left\{\alpha h(x ; \xi)+c x^{c-1}\left(1+x^{c}\right)^{-1}\right\},
$$

for $c, \alpha, \xi>0$ and $x \geq 0$. The cumulative distribution function (cdf) of the distribution is given by

$$
F(x ; c, \alpha, \beta)=1-\left(1+x^{c}\right)^{-1} e^{-\alpha H(x ; \xi)},
$$

for $c, \alpha, \xi>0$. Santos-Neto et al. (2014) proposed a new class of models called the Marshall-Olkin extended Weibull (MOEW) family of distributions based 
on the work by Marshall and Olkin (1997). The survival function is given as

$$
\bar{G}(x ; \alpha, \delta, \xi)=\frac{\delta \exp [-\alpha H(x ; \xi)]}{1-\bar{\delta} \exp [-\alpha H(x ; \xi)]},
$$

where $\bar{\delta}=1-\delta$ and for $\alpha, \delta>0$. The associated hazard rate reduces to

$$
h(x ; \alpha, \delta, \xi)=\frac{\delta h(x ; \xi)}{1-\bar{\delta} \exp [-\alpha H(x ; \xi)]}, \quad x \in \mathcal{D}, \alpha>0, \delta>0 .
$$

The corresponding pdf is given by

$$
g(x ; \alpha, \delta, \xi)=\frac{\delta \alpha h(x ; \xi) \exp [-\alpha H(x ; \xi)]}{[1-\bar{\delta} \exp [-\alpha H(x ; \xi)]]^{2}}, \quad x \in \mathcal{D}, \alpha>0, \delta>0,
$$

where $\delta=1-\bar{\delta}$. The general case of Marshall-Olkin Log-Logistic Extended Weibull (MOLLEW) family of distributions has survival function that is given by

$$
\bar{G}_{M O L L E W}(x ; c, \alpha, \delta, \xi)=\frac{\delta\left(1+x^{c}\right)^{-1} e^{-\alpha H(x ; \xi)}}{1-\bar{\delta}\left(1+x^{c}\right)^{-1} e^{-\alpha H(x ; \xi)}} .
$$

The pdf is given by

$$
g_{\text {MOLLEW }}(x ; c, \alpha, \delta, \xi)=\frac{\delta\left(1+x^{c}\right)^{-1} e^{-\alpha H(x ; \xi)}\left\{\alpha h(x ; \xi)+c x^{c-1}\left(1+x^{c}\right)^{-1}\right\}}{\left[1-\bar{\delta}\left(1+x^{c}\right)^{-1} e^{-\alpha H(x ; \xi)}\right]^{2}}
$$

for $c, \alpha, \xi, \delta>0$ and $x \geq 0$. The parameter $\alpha$ control the scale of the distribution and $\mathrm{c}$ and $\xi$ controls the shape of the distribution and $\delta$ is the tilt parameter. The hazard and reverse functions of the MOLLEW distribution are given by

$$
h_{M O L L E W}(x ; c, \alpha, \xi, \delta)=\frac{\alpha h(x ; \xi)+c x^{c-1}\left(1+x^{c}\right)^{-1}}{1-\bar{\delta}\left(1+x^{c}\right)^{-1} e^{-\alpha H(x ; \xi)}},
$$

and

$$
\tau_{M O L L E W}(x ; c, \alpha, \xi, \delta)=\frac{\alpha h(x ; \xi)+c x^{c-1}\left(1+x^{c}\right)^{-1}}{1-\left(1+x^{c}\right)^{-1} e^{-\alpha H(x ; \xi)}},
$$

for $c, \alpha, \xi, \delta>0$ and $x \geq 0$, respectively.

\subsection{Quantile Function}

The MOLLEW quantile function can be obtained by inverting $\bar{G}(x)=1-u$, where $G(x)=u, 0 \leq u \leq 1$, and

$$
\bar{G}_{M O L L E W}(x ; c, \alpha, \beta, \delta, \xi)=\frac{\delta\left(1+x^{c}\right)^{-1} e^{-\alpha H(x ; \xi)}}{1-\bar{\delta}\left(1+x^{c}\right)^{-1} e^{-\alpha H(x ; \xi)}} .
$$


The quantile function of the MOLLEW distribution is obtained by solving the non-linear equation

$$
\alpha H(x ; \xi)+\log \left(1+x^{c}\right)+\log \left[\frac{1-u}{\delta+(1-u) \bar{\delta}}\right]=0,
$$

using numerical methods. Consequently, random number can be generated based on equation (2.13).

\subsection{Expansion for the Density Function}

Using the generalized binomial expression

$$
(1-z)^{-k}=\sum_{j=0}^{\infty} \frac{\Gamma(k+j)}{\Gamma(k) j !} z^{j}, \quad \text { for }|z|<1,
$$

the MOLLEW pdf can be rewritten as follows:

$$
\begin{aligned}
g_{\text {MOLLEW }}(x) & =\sum_{j=0}^{\infty}(j+1) \delta \bar{\delta}^{j}\left[\left(1+x^{c}\right)^{-1} e^{-\alpha H(x ; \xi)}\right]^{j}\left(1+x^{c}\right)^{-1} e^{-\alpha H(x ; \xi)} \\
& \times\left\{\alpha h(x ; \xi)+c x^{c-1}\left(1+x^{c}\right)^{-1}\right\} \\
& =\sum_{j=0}^{\infty} \delta \bar{\delta}^{j}\left(1+x^{c}\right)^{-(j+1)-1} e^{-\alpha(j+1) H(x ; \xi)} \\
& \left.\times\left\{\alpha(j+1) h(x ; \xi)\left(1+x^{c}\right)+c(j+1) x^{c-1}\right)\right\} \\
& =\sum_{j=0}^{\infty} w(j, \delta) f_{B W}(x, c, j+1, \alpha(j+1), \xi)
\end{aligned}
$$

where $w(j, \delta)=\delta \bar{\delta}^{j}$ and $f_{B W}(x, c, j+1, \alpha(j+1), \xi)$ is the Burr XII Extended Weibull pdf with parameters for $c, \alpha(j+1), \xi, \delta>0$ and $x \geq 0$, respectively. Thus MOLLEW pdf can be written as a linear combination of Burr XII Extended Weibull density functions. The mathematical and statistical properties of the MOLLEW density function follows directly from those of the Burr XII Extended Weibull density function.

\section{Moments, Moment Generating Function and Conditional Moments}

In this section, moments, moment generating function and conditional moments are given for the MOLLEW distribution. The ${ }^{t h}$ moment of the MOLLEW 
distribution is given by

$$
\begin{aligned}
E\left(X^{r}\right) & =\int_{0}^{\infty} x^{r} g_{\text {MOLLEW }}(x) d x \\
& =\int_{0}^{\infty} x^{r} \delta\left(1+x^{c}\right)^{-1} e^{-\alpha H(x ; \xi)}\left\{\alpha h(x ; \xi)+c x^{c-1}\left(1+x^{c}\right)^{-1}\right\} \\
& \times\left[1-\bar{\delta}\left(1+x^{c}\right)^{-1} e^{-\alpha H(x ; \xi)]^{-2}} d x\right. \\
& =\int_{0}^{\infty} x^{r}\left[\sum_{j=0}^{\infty}(j+1) \delta \bar{\delta}^{j}\left[\left(1+x^{c}\right)^{-1} e^{-\alpha H(x ; \xi)}\right]^{j}\left(1+x^{c}\right)^{-1} e^{-\alpha H(x ; \xi)}\right. \\
& \left.\times\left\{\alpha h(x ; \xi)+c x^{c-1}\left(1+x^{c}\right)^{-1}\right\}\right] d x \\
& =\sum_{j=0}^{\infty} \delta \bar{\delta}^{j}\left[(\alpha(j+1)) \int_{0}^{\infty} x^{r}\left(1+x^{c}\right)^{-(j+1)} e^{-\alpha(j+1) H(x ; \xi)} h(x ; \xi) d x\right. \\
& +\left(c(j+1) \int_{0}^{\infty} x^{r+c-1}\left(1+x^{c}\right)^{-(j+1)-1} e^{-\alpha(j+1) H(x ; \xi)}\right] d x .
\end{aligned}
$$

Applying the power series expansion

$$
e^{-\alpha(j+1) H(x ; \xi)}=\sum_{i=0}^{\infty} \frac{(-1)^{i}[\alpha(j+1) H(x ; \xi)]^{i}}{i !},
$$

we have

$$
\begin{aligned}
E\left(X^{r}\right) & =\sum_{j=0}^{\infty} \delta \bar{\delta}^{j}\left[(\alpha(j+1)) \int_{0}^{\infty} x^{r}\left(1+x^{c}\right)^{-(j+1)} \sum_{i=0}^{\infty} \frac{(-1)^{i}[\alpha(j+1) H(x ; \xi)]^{i}}{i !} h(x ; \xi) d x\right. \\
& \left.+(c(j+1)) \int_{0}^{\infty} x^{r+c-1}\left(1+x^{c}\right)^{-(j+1)-1} \sum_{i=0}^{\infty} \frac{(-1)^{i}[\alpha(j+1) H(x ; \xi)]^{i}}{i !}\right] d x \\
& =\sum_{j=0}^{\infty} \sum_{i=0}^{\infty} \delta \bar{\delta}^{j} \frac{(-1)^{i}}{i !}(j+1)^{i+1}\left[\left(\alpha^{i+1}\right) \int_{0}^{\infty} x^{r}\left(1+x^{c}\right)^{-(j+1)} h(x ; \xi) H(x ; \xi)^{i} d x\right. \\
& \left.+\left(c \alpha^{i}\right) \int_{0}^{\infty} x^{r+c-1}\left(1+x^{c}\right)^{-(j+1)-1} H(x ; \xi)^{i}\right] d x .
\end{aligned}
$$




\subsection{Conditional Moments}

The $r^{\text {th }}$ conditional moment for MOLLEW distribution is given by

$$
\begin{aligned}
E\left(X^{r} \mid X>t\right) & =\frac{1}{\bar{G}_{\text {MOLLEW }}(t)} \int_{t}^{\infty} x^{r} g_{\text {MOLLEW }}(x) d x \\
& =\frac{1}{\overline{G_{M O L L E W}}(t)}\left[\sum_{j=0}^{\infty} \sum_{i=0}^{\infty} \delta \bar{\delta}^{j} \frac{(-1)^{i}}{i !}(j+1)^{i+1}\right. \\
& \times\left[\left(\alpha^{i+1}\right) \int_{t}^{\infty} x^{r}\left(1+x^{c}\right)^{-(j+1)} h(x ; \xi) H(x ; \xi)^{i} d x\right. \\
& \left.+\left(c \alpha^{i}\right) \int_{t}^{\infty} x^{r+c-1}\left(1+x^{c}\right)^{-(j+1)-1} H(x ; \xi)^{i}\right] d x
\end{aligned}
$$

Note that once $H(x ; \xi)$ is specified, the moment and conditional moments can be readily obtained.

\subsection{Bonferroni and Lorenz Curves}

In this subsection, we present Bonferroni and Lorenz curves. Bonferroni and Lorenz curves have applications not only in economics for the study income and poverty, but also in other fields such as reliability, demography, insurance and medicine. Bonferroni and Lorenz curves for the MOLLEW distribution are given by

$$
B(p)=\frac{1}{p \mu} \int_{0}^{q} x g_{M O L L E W}(x) d x=\frac{1}{p \mu}[\mu-T(q)],
$$

and

$$
L(p)=\frac{1}{\mu} \int_{0}^{q} x g_{M O L L E W}(x) d x=\frac{1}{\mu}[\mu-T(q)],
$$

respectively. The special cases for specified $H(x ; \xi)$ can be readily computed.

\section{Rényi Entropy}

The concept of entropy plays a vital role in information theory. The entropy of a random variable is defined in terms of its probability distribution and can be shown to be a good measure of randomness or uncertainty. In this section, we present Rényi entropy for the MOLLEW distribution. Rényi entropy is an extension of Shannon entropy. Rényi entropy is defined to be

$$
I_{R}(v)=\frac{1}{1-v} \log \left(\int_{0}^{\infty}[g(x ; c, \alpha, \xi, \delta)]^{v} d x\right), v \neq 1, v>0 .
$$


Rényi entropy tends to Shannon entropy as $v \rightarrow 1$. Note that $[g(x ; c, \alpha, \xi, \theta)]^{v}=$ $g^{v}(x)$ can be written as

$$
\begin{aligned}
g^{v}(x) & =\left[\delta\left(1+x^{c}\right)^{-1} e^{-\alpha H(x ; \xi)}\left\{\alpha h(x ; \xi)+c x^{c-1}\left(1+x^{c}\right)^{-1}\right\}\right. \\
& \left.\times\left[1-\bar{\delta}\left(1+x^{c}\right)^{-1} e^{-\alpha H(x ; \xi)}\right]^{-2}\right]^{v} \\
& =\sum_{j=0}^{\infty} \sum_{p=0}^{\infty} \sum_{w=0}^{v}\left(\begin{array}{c}
v \\
w
\end{array}\right)\left(\frac{(-1)^{p} \Gamma(j+2 v) \delta^{v} \bar{\delta}^{j}(v+j)^{p}}{\Gamma(2 v) j ! p !}\right) \\
& \times \alpha^{p+v-w}\left(1+x^{c}\right)^{-j-v-w} x^{c w-w} h(x ; \xi)^{v-w} H(x ; \xi)^{p}
\end{aligned}
$$

Thus,

$$
\begin{aligned}
\int_{0}^{\infty} g^{v}(x) d x & =\sum_{j=0}^{\infty} \sum_{p=0}^{\infty} \sum_{w=0}^{v}\left(\begin{array}{c}
v \\
w
\end{array}\right)\left(\frac{(-1)^{p} \Gamma(j+2 v) \delta^{v} \bar{\delta}^{j}(v+j)^{p}}{\Gamma(2 v) j ! p !}\right) \\
& \times \alpha^{p+v-w} \int_{0}^{\infty}\left(1+x^{c}\right)^{-j-v-w} x^{c w-w} h(x ; \xi)^{v-w} H(x ; \xi)^{p} d x .
\end{aligned}
$$

Consequently, Rényi entropy is given by

$$
\begin{aligned}
I_{R}(v) & =\left(\frac{1}{1-v}\right) \log \left(\sum_{j=0}^{\infty} \sum_{p=0}^{\infty} \sum_{w=0}^{v}\left(\begin{array}{c}
v \\
w
\end{array}\right)\left(\frac{(-1)^{p} \Gamma(j+2 v) \delta^{v} \bar{\delta}^{j}(v+j)^{p}}{\Gamma(2 v) j ! p !}\right)\right. \\
& \left.\times \alpha^{p+v-w} \int_{0}^{\infty}\left(1+x^{c}\right)^{-j-v-w} x^{c w-w} h(x ; \xi)^{v-w} H(x ; \xi)^{p} d x\right)
\end{aligned}
$$

for $v \neq 1$, and $v>0$.

\section{Maximum Likelihood Estimation}

Let $X \sim \operatorname{MOLLEW}(c, \alpha, \xi, \delta)$ and $\boldsymbol{\Delta}=(c, \alpha, \xi, \delta)^{T}$ be the parameter vector. The log-likelihood function $\ell=\ell(\boldsymbol{\Delta})$ based on a random sample of size $n$ is given by

$$
\begin{aligned}
\ell(\boldsymbol{\Delta}) & =n \log \delta-\sum_{i=0}^{n} \log \left(1+x_{i}^{c}\right)-\alpha \sum_{i=0}^{n} H\left(x_{i} ; \xi\right) \\
& +\sum_{i=0}^{n} \log \left(\alpha h\left(x_{i} ; \xi\right)+c x_{i}^{c-1}\left(1+x_{i}^{c}\right)^{-1}\right)-2 \sum_{i=0}^{n} \log \left(1-\bar{\delta}\left(1+x_{i}^{c}\right)^{-1} e^{-\alpha H\left(x_{i} ; \xi\right)}\right) .
\end{aligned}
$$

The elements of the score vector $U=\left(\frac{\partial \ell}{\partial \delta}, \frac{\partial \ell}{\partial \alpha}, \frac{\partial \ell}{\partial \xi_{k}}, \frac{\partial \ell}{\partial c}\right)$ are given by

$$
\frac{\partial \ell}{\partial \delta}=\frac{n}{\delta}-2 \sum_{i=0}^{n} \frac{\left(1+x_{i}^{c}\right)^{-1} e^{-\alpha H\left(x_{i} ; \xi\right)}}{1-\bar{\delta}\left(1+x_{i}^{c}\right)^{-1} e^{-\alpha H\left(x_{i} ; \xi\right)}},
$$




$$
\begin{gathered}
\frac{\partial \ell}{\partial \alpha}=-\sum_{i=0}^{n} H\left(x_{i} ; \xi\right)+\sum_{i=0}^{n} \frac{\alpha h\left(x_{i} ; \xi\right)}{\alpha h\left(x_{i} ; \xi\right)+c x_{i}{ }^{c-1}\left(1+x_{i}^{c}\right)^{-1}} \\
-2 \bar{\delta} \sum_{i=0}^{n} \frac{H\left(x_{i} ; \xi\right)\left(1+x_{i}^{c}\right)^{-1} e^{-\alpha H\left(x_{i} ; \xi\right)}}{1-\bar{\delta}\left(1+x_{i}^{c}\right)^{-1} e^{-\alpha H\left(x_{i} ; \xi\right)}} \\
\frac{\partial \ell}{\partial \xi_{k}}=-\sum_{i=0}^{n} \alpha \frac{\partial H\left(x_{i} ; \xi\right)}{\partial \xi_{k}}+\alpha \sum_{i=0}^{n} \frac{\partial h\left(x_{i} ; \xi\right)}{\partial \xi_{k}} \frac{1}{\alpha h\left(x_{i} ; \xi\right)+c x_{i}{ }^{c-1}\left(1+x_{i}^{c}\right)^{-1}} \\
-2 \bar{\delta} \alpha \sum_{i=0}^{n} \frac{\partial H\left(x_{i} ; \xi\right)}{\partial \xi_{k}} \frac{\left(1+x_{i}^{c}\right)^{-1} e^{-\alpha H\left(x_{i} ; \xi\right)}}{1-\bar{\delta}\left(1+x_{i}^{c}\right)^{-1} e^{-\alpha H\left(x_{i} ; \xi\right)}},
\end{gathered}
$$

and

$$
\begin{aligned}
\frac{\partial \ell}{\partial c} & =-\sum_{i=0}^{n} \frac{x^{c} \log x_{i}}{\left(1+x_{i}^{c}\right)}-2 \alpha \sum_{i=0}^{n} \frac{\bar{\delta}\left(1+x_{i}^{c}\right)^{-1} e^{-\alpha H\left(x_{i} ; \xi\right)}}{1-\bar{\delta}\left(1+x_{i}^{c}\right)^{-1} e^{-\alpha H\left(x_{i} ; \xi\right)} \log x_{i}} \\
& +\frac{x_{i}^{c-1}\left(1+x_{i}^{c}\right)^{-1}-c x_{i}^{2 c-1}\left(1+x_{i}^{c}\right)^{-2} \log x_{i}+c x_{i}^{c-1}\left(1+x_{i}^{c}\right)^{-1} \log x_{i}}{\alpha h\left(x_{i} ; \xi\right)+c x_{i}{ }^{c-1}\left(1+x_{i}^{c}\right)^{-1}} .
\end{aligned}
$$

The equations are obtained by setting the above partial derivatives to zero are not in closed form and the values of the parameters $c, \alpha, \xi$ and $\delta$ must be found via iterative methods. The maximum likelihood estimates of the parameters, denoted by $\hat{\boldsymbol{\Delta}}$ is obtained by solving the nonlinear equation $\left(\frac{\partial \ell}{\partial c}, \frac{\partial \ell}{\partial \alpha}, \frac{\partial \ell}{\partial \xi_{k}}, \frac{\partial \ell}{\partial \delta}\right)^{T}=\mathbf{0}$, using a numerical method such as Newton-Raphson procedure. The Fisher information matrix is given by $\mathbf{I}(\boldsymbol{\Delta})=\left[\mathbf{I}_{\theta_{i}, \theta_{j}}\right]_{4 X 4}=$ $E\left(-\frac{\partial^{2} \ell}{\partial \theta_{i} \partial \theta_{j}}\right), i, j=1,2,3,4$, can be numerically obtained by MATLAB or NLMIXED in SAS or R software. The total Fisher information matrix $n \mathbf{I}(\boldsymbol{\Delta})$ can be approximated by

$$
\mathbf{J}_{n}(\hat{\boldsymbol{\Delta}}) \approx\left[-\left.\frac{\partial^{2} \ell}{\partial \theta_{i} \partial \theta_{j}}\right|_{\boldsymbol{\Delta}=\hat{\boldsymbol{\Delta}}}\right]_{4 X 4}, \quad i, j=1,2,3,4 .
$$

For a given set of observations, the matrix given in equation (5.1) is obtained after the convergence of the Newton-Raphson procedure via NLMIXED in SAS or R software.

\section{Marshal-Olkin Log-Logistic Weibull (MOLLW) Distribution}

Now consider the Log-Logistic-Weibull distribution. This distribution is obtained via the use of competing risk model and is given by combining both the 
Log-Logistic and Weibull distribution. In this case, we take $H(x ; \xi)=x^{\beta}$ in the MOLLEW distribution. The MOLLW cdf is given by

$$
F(x ; c, \alpha, \beta)=1-\left(1+x^{c}\right)^{-1} e^{-\alpha x^{\beta}},
$$

for $c, \alpha, \beta>0$. The corresponding pdf is given by

$$
f(x ; c, \alpha, \beta)=e^{-\alpha x^{\beta}}\left(1+x^{c}\right)^{-1}\left\{\alpha \beta x^{\beta-1}+c x^{c-1}\left(1+x^{c}\right)^{-1}\right\}
$$

for $c, \alpha, \beta>0$ and $x \geq 0$. Recall the extended case, Marshall-Olkin Log Logistic Extended Weibull (MOLLEW) distribution has survival function given by

$$
\bar{G}_{M O L L E W}(x ; c, \alpha, \beta, \delta, \xi)=\frac{\delta\left(1+x^{c}\right)^{-1} e^{-\alpha H(x ; \xi)}}{1-\bar{\delta}\left(1+x^{c}\right)^{-1} e^{-\alpha H(x ; \xi)}}
$$

with corresponding pdf given by

$$
g_{\text {MOLLEW }}(x ; c, \alpha, \beta, \delta, \xi)=\frac{\delta\left(1+x^{c}\right)^{-1} e^{-\alpha H(x ; \xi)}\left\{\alpha \beta x^{\beta-1}+c x^{c-1}\left(1+x^{c}\right)^{-1}\right\}}{\left[1-\bar{\delta}\left(1+x^{c}\right)^{-1} e^{-\alpha x^{\beta}}\right]^{2}} .
$$

In this section, we study a special case of the family, namely MarshallOlkin Log-Logistic Weibull (MOLLW) distribution, by taking $H(x ; \xi)=x^{\beta}$. The MOLLW survival function is given by

$$
\bar{G}_{M O L L W}(x ; c, \alpha, \beta, \delta)=\frac{\delta\left(1+x^{c}\right)^{-1} e^{-\alpha x^{\beta}}}{1-\bar{\delta}\left(1+x^{c}\right)^{-1} e^{-\alpha x^{\beta}}} .
$$

The corresponding pdf is given by

$$
g_{M O L L W}(x ; c, \alpha, \beta, \delta)=\frac{\delta\left(1+x^{c}\right)^{-1} e^{-\alpha x^{\beta}}\left\{\alpha \beta x^{\beta-1}+c x^{c-1}\left(1+x^{c}\right)^{-1}\right\}}{\left[1-\bar{\delta}\left(1+x^{c}\right)^{-1} e^{-\alpha x^{\beta}}\right]^{2}}
$$

for $c, \alpha, \beta, \delta>0$ and $x \geq 0$. Note that the parameter $\alpha$ control the scale of the distribution, $\mathrm{c}$ and $\beta$ controls the shape of the distribution and $\delta$ is the tilt parameter. The plots of the MOLLW pdf is given in Figure 6.1. The plots shows that the pdf can be L-shaped, decreasing, unimodal depending on selected parameter values.

\subsection{Quantile Function}

The quantile function of the MOLLW distribution is obtained by solving the non-linear equation

$$
\alpha x^{\beta}+\log \left(1+x^{c}\right)+\log \left[\frac{1-u}{\delta+(1-u) \bar{\delta}}\right]=0 .
$$




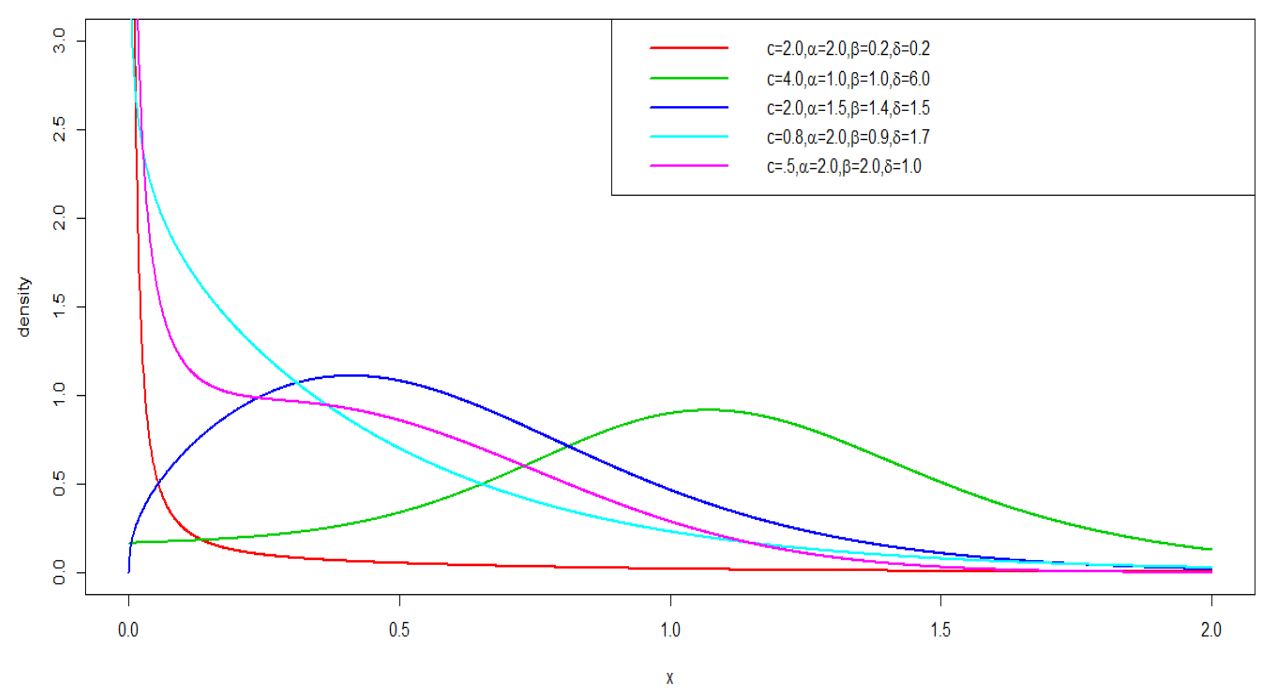

Figure 6.1: Plots of MOLLW PDF

The quantile function of the MOLLW distribution is obtained by solving the non-linear equation using numerical methods. Consequently, random number can be generated based on equation 6.7). Table 6.1 lists the quantile for selected values of the parameters of the MOLLW distribution.

Table 6.1: MOLLW Quantile for Selected Values

\begin{tabular}{cccccc}
\hline & \multicolumn{5}{c}{$(c, \alpha, \beta, \delta)$} \\
\cline { 2 - 6 }$u$ & $(2.0,2.0,0.5,0.5,0.1)$ & $(2,0.5,2.0,0.5)$ & $(0.5,0.5,1.5,1.5)$ & $(0.4,1.0,0.4,0.7)$ & $(1.0,1.5,2.0,2.0)$ \\
\hline 0.1 & 0.00072 & 0.19099 & 0.0269 & $0 . .00027$ & 0.17021 \\
0.2 & $0 . .0347$ & 0.28389 & 0.12019 & 0.00195 & 0.30481 \\
0.3 & 0.00943 & 0.36744 & 0.27768 & 0.00675 & 0.42192 \\
0.4 & 0.02060 & 0.45170 & 0.48049 & 0.01798 & 0.53029 \\
0.5 & 0.04076 & 0.54273 & 0.71626 & 0.04229 & 0.63584 \\
0.6 & 0.07666 & 0.64741 & 0.98551 & 0.09450 & 0.74393 \\
0.7 & 0.14185 & 0.77644 & 1.30194 & 0.21219 & 0.86148 \\
0.8 & 0.26549 & 0.95214 & 1.70267 & 0.51385 & 1.00116 \\
0.9 & 1.53053 & 1.24431 & 2.30566 & 1.57612 & 1.19901 \\
\hline
\end{tabular}




\subsection{Hazard and Reverse Hazard Functions}

The hazard and reverse functions of the MOLLW distribution are given by

$$
h_{M O L L W}(x ; c, \alpha, \beta, \delta)=\frac{\alpha \beta x^{\beta-1}+c x^{c-1}\left(1+x^{c}\right)^{-1}}{1-\bar{\delta}\left(1+x^{c}\right)^{-1} e^{-\alpha x^{\beta}}},
$$

and

$$
\tau_{M O L L W}(x ; c, \alpha, \beta, \delta)=(x ; c, \alpha, \beta, \delta)=\frac{\alpha \beta x^{\beta-1}+c x^{c-1}\left(1+x^{c}\right)^{-1}}{1-\left(1+x^{c}\right)^{-1} e^{-\alpha x^{\beta}}},
$$

for $c, \alpha, \beta, \delta>0$ and $x \geq 0$, respectively.

Plots of the MOLLW hazard function are given in Figure 6.2. The plots shows that the hazard function of the MOLLW can either be decreasing, bathtub followed by upside down bathtub or increasing-decreasing depending on selected parameter values.

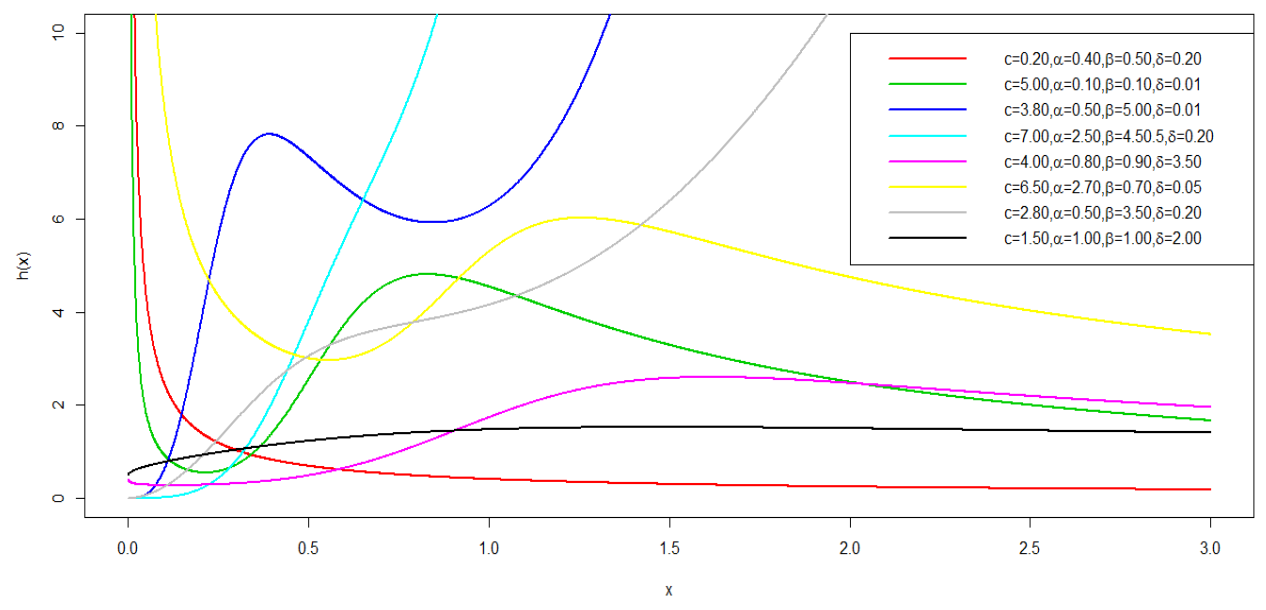

Figure 6.2: Plots of MOLLW Hazard

\subsection{Some Sub-models}

There are several new as well as well known distributions that can be obtained from the MOLLW distribution. The sub-models include the following distributions:

- When $\delta=1$, we obtain the Log-Logistic Weibull (LLW) distribution.

- When $\beta=2$, we obtain the Marshall-Olkin Log-Logistic Rayleigh (MOLLR) distribution. 
- If $\alpha \rightarrow 0^{+}$, we obtain the Marshall-Olkin Log-Logistic (MOLL) distribution.

- If $c=1$, then the Marshall-Olkin Log-Logistic Weibull distribution reduces to the 3 parameter distribution with survival function

$$
\bar{G}_{M O L L W}(x ; \alpha, \delta, \beta)=\frac{\delta(1+x)^{-1} e^{-\alpha x^{\beta}}}{1-\bar{\delta}(1+x)^{-1} e^{-\alpha x^{\beta}}} .
$$

- If $c=1$ and $\beta=1$, then the Marshall-Olkin Log-Logistic Weibull distribution reduces to the 2 parameter distribution with survival function

$$
\bar{G}_{M O L L W}(x ; \alpha, \delta)=\frac{\delta(1+x)^{-1} e^{-\alpha x}}{1-\bar{\delta}(1+x)^{-1} e^{-\alpha x}} .
$$

- If $c=1$ and $\beta=2$, then the Marshall-Olkin Log-Logistic Weibull distribution reduces to the 2 parameter distribution with survival function given by

$$
\bar{G}_{M O L L W}(x ; \alpha, \delta)=\frac{\delta(1+x)^{-1} e^{-\alpha x^{2}}}{1-\bar{\delta}(1+x)^{-1} e^{-\alpha x^{2}}} .
$$

\subsection{Expansion for the Density Function}

Using the generalized binomial expression

$$
(1-z)^{-k}=\sum_{j=0}^{\infty} \frac{\Gamma(k+j)}{\Gamma(k) j !} z^{j}, \quad \text { for }|z|<1,
$$

the MOLLW pdf can be rewritten as follows:

$$
g_{M O L L W}(x)=\sum_{j=0}^{\infty} w(j, \delta) f_{B W}(x, c, j+1, \alpha(j+1), \beta),
$$

where $w(j, \delta)=\delta \bar{\delta}^{j}$ and $f_{B W}(x, c, j+1, \alpha(j+1), \beta)$ is the Burr XII Weibull pdf with parameters for $c>0, j+1>0, \alpha(j+1)>0$, and $\beta>0$ respectively. Thus MOLLW pdf can be written as a linear combination of Burr XII-Weibull density functions. The mathematical and statistical properties of the MOLLW distribution follows directly from those of the Burr XII Weibull density function. 


\subsection{Moments, Moment Generating Function and Conditional Moments}

In this section, moments, moment generating function and conditional moments are given for the MOLLW distribution. The $r^{\text {th }}$ moment of the MOLLW distribution is given by

$$
\begin{aligned}
E\left(X^{r}\right) & =\sum_{j=0}^{\infty} \delta \bar{\delta}^{j}\left[(\alpha(j+1) \beta) \int_{0}^{\infty} x^{r+\beta-1}\left(1+x^{c}\right)^{-(j+1)} e^{-\alpha(j+1) x^{\beta}} d x\right. \\
& +\left(c(j+1) \int_{0}^{\infty} x^{r+c-1}\left(1+x^{c}\right)^{-(j+1)-1} e^{-\alpha(j+1) x^{\beta}}\right] d x .
\end{aligned}
$$

Applying the power series expansion

$$
e^{-\alpha(j+1) x^{\beta}}=\sum_{i=0}^{\infty} \frac{(-1)^{i}\left[\alpha(j+1) x^{\beta}\right]^{i}}{i !}
$$

we have

$$
\begin{aligned}
E\left(X^{r}\right) & =\sum_{j=0}^{\infty} \delta \bar{\delta}^{j}\left[(\alpha(j+1) \beta) \int_{0}^{\infty} x^{r+\beta-1}\left(1+x^{c}\right)^{-(j+1)} \sum_{i=0}^{\infty} \frac{(-1)^{i}\left[\alpha(j+1) x^{\beta}\right]^{i}}{i !} d x\right. \\
& \left.+(c(j+1)) \int_{0}^{\infty} x^{r+c-1}\left(1+x^{c}\right)^{-(j+1)-1} \sum_{i=0}^{\infty} \frac{(-1)^{i}\left[\alpha(j+1) x^{\beta}\right]^{i}}{i !}\right] d x \\
& =\sum_{j=0}^{\infty} \sum_{i=0}^{\infty} \delta \bar{\delta}^{j} \frac{(-1)^{i}}{i !}(j+1)^{i+1}\left[\left(\alpha^{i+1} \beta\right) \int_{0}^{\infty} x^{r+\beta+\beta i-1}\left(1+x^{c}\right)^{-(j+1)} d x\right. \\
& \left.+\left(c \alpha^{i}\right) \int_{0}^{\infty} x^{r+c+\beta i-1}\left(1+x^{c}\right)^{-(j+1)-1}\right] d x .
\end{aligned}
$$

Let $y=\left(1+x^{c}\right)^{-1}$, then $x=\left(\frac{1-y}{y}\right)^{\frac{1}{c}}$, and $d x=c^{-1} y^{-2}(1-y)^{\frac{1}{c}-1} y^{1-\frac{1}{c}} d y$, 
so that

$$
\begin{aligned}
E\left(X^{r}\right) & =\sum_{j=0}^{\infty} \sum_{i=0}^{\infty} \delta \bar{\delta}^{j} \frac{(-1)^{i}}{i !}(j+1)^{i+1}\left[\left(\alpha^{i+1} \beta\right)\right. \\
& \times \int_{0}^{1}\left(\left(\frac{1-y}{y}\right)^{\frac{1}{c}}\right)^{r+\beta+\beta i-1} y^{j+1} y^{-2} c^{-1}(1-y)^{\frac{1}{c}-1} y^{1-\frac{1}{c}} d y \\
& \left.+\left(c \alpha^{i}\right) \int_{0}^{1}\left(\left(\frac{1-y}{y}\right)^{\frac{1}{c}}\right)^{r+c+\beta i-1} y^{j+2} y^{-2} c^{-1}(1-y)^{\frac{1}{c}-1} y^{1-\frac{1}{c}}\right] d y \\
& =\sum_{j=0}^{\infty} \sum_{i=0}^{\infty} \delta \bar{\delta}^{j} \frac{(-1)^{i}}{i !}(j+1)^{i+1}\left[\left(c^{-1} \alpha^{i+1} \beta\right) \int_{0}^{1} y^{-\frac{r}{c}-\frac{\beta i}{c}-\frac{\beta}{c}+j}(1-y)^{-\frac{r}{c}+\frac{\beta i}{c}+\frac{\beta}{c}-1} d y\right. \\
& \left.+\alpha^{i} \int_{0}^{1} y^{-\frac{r}{c}-\frac{\beta i}{c}+j}(1-y)^{-\frac{r}{c}+\frac{\beta i}{c}} d y\right] \\
& =\sum_{j=0}^{\infty} \sum_{i=0}^{\infty} \delta \bar{\delta}^{j} \frac{(-1)^{i}}{i !}(j+1)^{i+1}\left[( c ^ { - 1 } \alpha ^ { i + 1 } \beta ) B \left(j-\frac{1}{c}(r+\beta i+\beta-c), \frac{1}{c}(r+\beta i+\beta)\right.\right. \\
& +\alpha^{i} B\left(j-\frac{1}{c}(r+\beta i-c), \frac{1}{c}(r+\beta i-c)\right],
\end{aligned}
$$

where $B(a, b)=\int_{0}^{1} t^{a-1}(1-t)^{b-1} d t$ is the complete beta function. The moment generating function (MGF) of the MOLLW distribution is given by $M_{X}(t)=$ $E\left(e^{t X}\right)=\sum_{n=0}^{\infty} \frac{t^{n}}{n !} E\left(X^{n}\right)$, where $E\left(X^{n}\right)$ is given above.

Table 6.2 lists the first five moments together with the standard deviation (SD or $\sigma$ ), coefficient of variation (CV), coefficient of skewness (CS) and coefficient of kurtosis $(\mathrm{CK})$ of the MOLLW distribution for selected values of the parameters, by fixing $\beta=1.5$ and $\delta=1.5$. Table 6.3 lists the first five moments, SD, CV, CS and CK of the MOLLW distribution for selected values of the parameters, by fixing $c=1.0$ and $\alpha=1.5$. These values can be determined numerically using $\mathrm{R}$ and MATLAB. The SD, CV, CS and CK are given by $\sigma=\sqrt{\mu_{2}^{\prime}-\mu^{2}}$

$$
\begin{gathered}
C V=\frac{\sigma}{\mu}=\frac{\sqrt{\mu_{2}^{\prime}-\mu^{2}}}{\mu}=\sqrt{\frac{\mu_{2}^{\prime}}{\mu^{2}}-1} \\
C S=\frac{E\left[(X-\mu)^{3}\right]}{\left[E(X-\mu)^{2}\right]^{3 / 2}}=\frac{\mu_{3}^{\prime}-3 \mu \mu_{2}^{\prime}+2 \mu^{3}}{\left(\mu_{2}^{\prime}-\mu^{2}\right)^{3 / 2}},
\end{gathered}
$$

and

$$
C K=\frac{E\left[(X-\mu)^{4}\right]}{\left[E(X-\mu)^{2}\right]^{2}}=\frac{\mu_{4}^{\prime}-4 \mu \mu_{3}^{\prime}+6 \mu^{2} \mu_{2}^{\prime}-3 \mu^{4}}{\left(\mu_{2}^{\prime}-\mu^{2}\right)^{2}},
$$

respectively.

Plots of the skewness and kurtosis for selected choices of the parameter $\beta$ as a function of $c$ as well as for some selected choices of $c$ as a function of $\beta$ are 
displayed in figure 6.3. The plots show that the skewness and kurtosis depend on the shape parameters $c$ and $\beta$.

Plotof Skemess $(0=1.5$ and $\delta=2.0)$

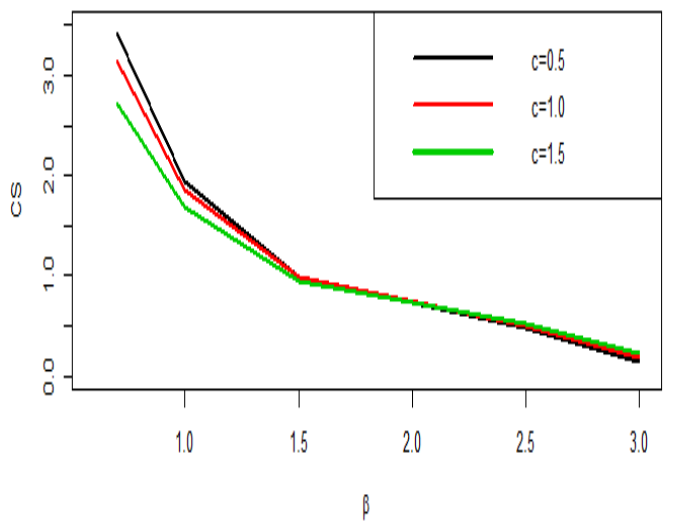

Plotof Kurtosis (0:1.5and 8=2.0)

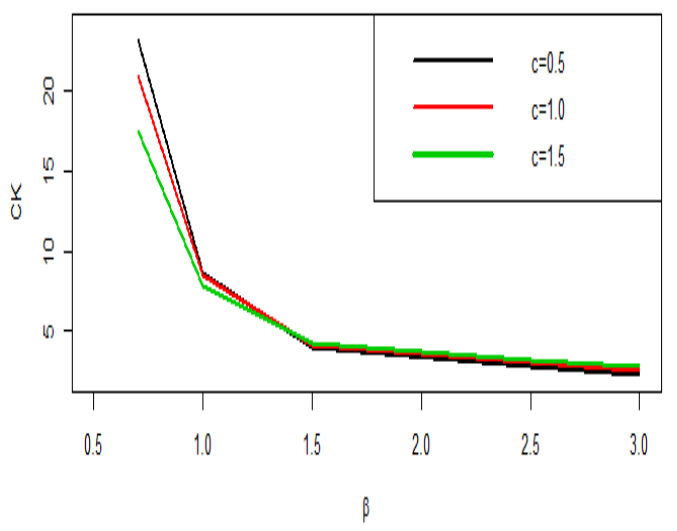

Plotof Skemness $(0=2.5$ and $8=2.5)$

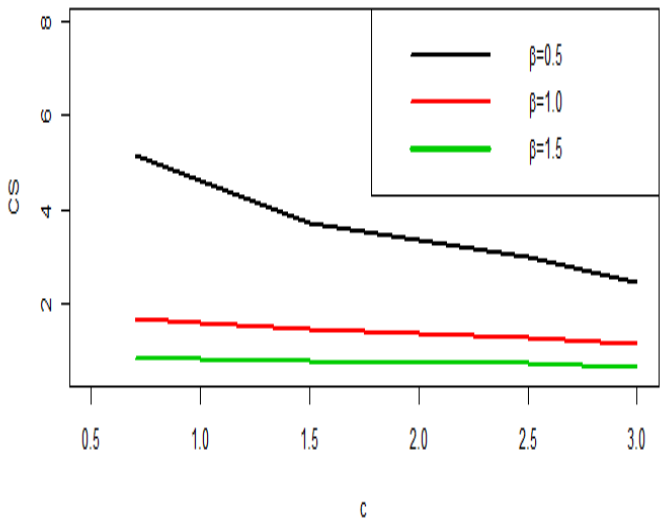

Plotof Kurtosis (a=2.5.5and 8=2.5)

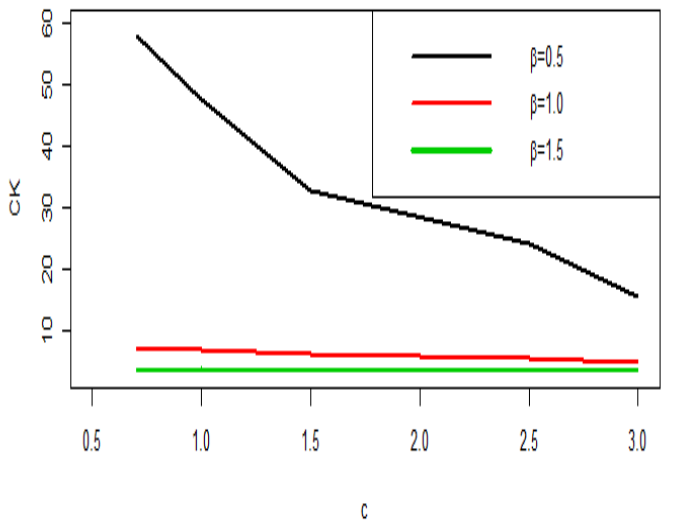

Figure 6.3: Plots of skewness and kurtosis for selected parameter values 
Table 6.2: Moments of the MOLLW distribution for some parameter values; $\beta=1.5$ and $\delta=1.5$.

\begin{tabular}{lrrrr}
\hline$\mu_{s}^{\prime}$ & $c=0.5, \alpha=0.5$ & $c=0.5, \alpha=0.5$ & $c=2.0, \alpha=2.0$ & $c=2.0, \alpha=2.0$ \\
\hline$\mu_{1}^{\prime}$ & 0.9731853 & 0.4508166 & 0.9680721 & 0.5463952 \\
$\mu_{2}^{\prime}$ & 1.8408579 & 0.3554505 & 1.3474524 & 0.4096721 \\
$\mu_{3}^{\prime}$ & 4.6181391 & 0.3637649 & 2.4537575 & 0.3797837 \\
$\mu_{4}^{\prime}$ & 14.0188390 & 0.4463212 & 5.5553411 & 0.4151282 \\
$\mu_{5}^{\prime}$ & 49.2885587 & 0.6310007 & 15.0885743 & 0.5203804 \\
$\mathrm{SD}$ & 0.9453931 & 0.3901472 & 0.6405379 & 0.3333532 \\
$\mathrm{CV}$ & 0.9714420 & 0.8654233 & 0.6616635 & 0.6100954 \\
$\mathrm{CS}$ & 1.2864943 & 1.1160837 & 1.3506110 & 0.9314745 \\
$\mathrm{CK}$ & 4.7713115 & 4.3110027 & 5.9140779 & 4.1727749 \\
\hline
\end{tabular}

Table 6.3: Moments of the MOLLW distribution for some parameter values; $c=1.0$ and $\alpha=1.5$.

\begin{tabular}{rrrrr}
\hline$\mu_{s}^{\prime}$ & $\beta=0.7, \delta=1.2$ & $\beta=1.0, \delta=1.0$ & $\beta=2.5, \delta=2.5$ & $\beta=1.5, \delta=0.4$ \\
\hline$\mu_{1}^{\prime}$ & 0.4753178 & 0.4482567 & 0.73153814 & 0.3171233 \\
$\mu_{2}^{\prime}$ & 0.6638554 & 0.4368200 & 0.66668141 & 0.2069297 \\
$\mu_{3}^{\prime}$ & 1.8042518 & 0.6781033 & 0.68452169 & 0.2027406 \\
$\mu_{4}^{\prime}$ & 8.0305974 & 1.4662326 & 0.76541391 & 0.2587302 \\
$\mu_{5}^{\prime}$ & 52.6285665 & 4.0931352 & 0.91608242 & 0.3988813 \\
$\mathrm{SD}$ & 0.6617616 & 0.4856809 & 0.36267529 & 0.3261326 \\
$\mathrm{CV}$ & 1.3922510 & 1.0834884 & 0.49577086 & 1.0284094 \\
$\mathrm{CS}$ & 3.7004265 & 2.3639005 & 0.09172126 & 2.0081229 \\
$\mathrm{CK}$ & 27.8806818 & 11.7875287 & 2.53674353 & 8.4925412 \\
\hline
\end{tabular}

\subsection{Conditional Moments}

The $r^{\text {th }}$ conditional moment for MOLLW is given by

$$
\begin{aligned}
E\left(X^{r} \mid X>t\right) & =\frac{1}{\bar{G}_{M O L L W}(t)} \int_{t}^{\infty} x^{r} g_{M O L L W}(x) d x \\
& =\frac{1}{\bar{G}_{M O L L W}(t)} \int_{t}^{\infty} x^{r} \delta\left(1+x^{c}\right)^{-1} e^{-\alpha x^{\beta}}\left\{\alpha \beta x^{\beta-1}+c x^{c-1}\left(1+x^{c}\right)^{-1}\right\} \\
& \times\left(1-\bar{\delta}\left(1+x^{c}\right)^{-1} e^{-\alpha x^{\beta}}\right)^{-2} d x .
\end{aligned}
$$


Let $y=\left(1+x^{c}\right)^{-1}$, then $x=\left(\frac{1-y}{y}\right)^{\frac{1}{c}}$, and $d x=c^{-1} y^{-2}(1-y)^{\frac{1}{c}-1} y^{1-\frac{1}{c}} d y$, . Now

$$
\begin{aligned}
E\left(X^{r} \mid X>t\right) & =\frac{1}{\bar{G}_{M O L L W}(t)}\left[\sum_{j=0}^{\infty} \sum_{i=0}^{\infty} \delta \bar{\delta}^{j} \frac{(-1)^{i}}{i !}(j+1)^{i+1}\right. \\
& \times\left[( c ^ { - 1 } \alpha ^ { i + 1 } \beta ) B _ { ( 1 + t ^ { c } ) ^ { - 1 } } \left(j-\frac{1}{c}(r+\beta i+\beta-c), \frac{1}{c}(r+\beta i+\beta)\right.\right. \\
& \left.+\alpha^{i} B_{\left(1+t^{c}\right)^{-1}}\left(j-\frac{1}{c}(r+\beta i-c)\right), \frac{1}{c}(r+\beta i-c)\right],
\end{aligned}
$$

where $B_{y}(a, b)=\int_{0}^{y} x^{a-1}(1-x)^{b-1} d x$ is the incomplete beta function. The mean residual lifetime function of the MOLLW distribution is given by $E(X \mid X>$ $t)-t$

\subsection{Bonferroni and Lorenz Curves}

In this subsection, we present Bonferroni and Lorenz Curves. Bonferroni and Lorenz curves have applications not only in economics for the study income and poverty, but also in other fields such as reliability, demography, insurance and medicine. Bonferroni and Lorenz curves for the MOLLW distribution are given by

$$
B(p)=\frac{1}{p \mu} \int_{0}^{q} x g_{M O L L W}(x) d x=\frac{1}{p \mu}[\mu-T(q)]
$$

and

$$
L(p)=\frac{1}{\mu} \int_{0}^{q} x g_{M O L L W}(x) d x=\frac{1}{\mu}[\mu-T(q)],
$$

respectively, where $T(q)=\int_{q}^{\infty} x g(x) d x$, is given by

$$
\begin{aligned}
\tau(q)=\int_{q}^{\infty} x g_{M O L L W}(x) d x & =\sum_{j=0}^{\infty} \sum_{i=0}^{\infty} \delta \bar{\delta}^{j} \frac{(-1)^{i}}{i !}(j+1)^{i+1}\left(c^{-1} \alpha^{i+1} \beta\right) \\
& \times\left[B _ { ( 1 + q ^ { c } ) ^ { - 1 } } \left(j-\frac{1}{c}(r+\beta i+\beta-c), \frac{1}{c}(r+\beta i+\beta) \alpha^{i}\right.\right. \\
& +B_{\left(1+q^{c}\right)^{-1}}\left(j-\frac{1}{c}(r+\beta i-c), \frac{1}{c}(r+\beta i-c)\right]
\end{aligned}
$$

and $q=G^{-1}(p), 0 \leq p \leq 1$

\subsection{Rényi Entropy}

The concept of entropy plays a vital role in information theory. The entropy of a random variable is defined in terms of its probability distribution and can be shown to be a good measure of randomness or uncertainty. In this subsection, 
Rényi entropy of the MOLLW distribution is derived. An entropy is a measure of uncertainty or variation of a random variable. Rényi entropy is an extension of Shannon entropy. Note that $[g(x ; c, \alpha, \beta, \theta)]^{v}=g^{v}(x)$ can be written as

$$
\begin{aligned}
g^{v}(x) & =\left[\delta\left(1+x^{c}\right)^{-1} e^{-\alpha x^{\beta}}\left\{\alpha \beta x^{\beta-1}+c x^{c-1}\left(1+x^{c}\right)^{-1}\right\}\left[1-\bar{\delta}\left(1+x^{c}\right)^{-1} e^{-\alpha x^{\beta}}\right]^{-2}\right]^{v} \\
& =\sum_{j=0}^{\infty} \sum_{p=0}^{\infty} \sum_{w=0}^{v}\left(\begin{array}{c}
v \\
w
\end{array}\right)\left(\frac{(-1)^{p} \Gamma(j+2 v) \delta^{v} \bar{\delta}^{j}(v+j)^{p}}{\Gamma(2 v) j ! p !}\right) \\
& \times \alpha^{p+v-w} \beta^{v-w} c^{w}\left(1+x^{c}\right)^{-j-v-w} x^{\beta p-\beta w+\beta v+c w-v} .
\end{aligned}
$$

Now,

$$
\begin{aligned}
\int_{0}^{\infty} g^{v}(x) d x & =\int_{0}^{\infty} \sum_{j=0}^{\infty} \sum_{p=0}^{\infty} \sum_{w=0}^{v}\left(\begin{array}{c}
v \\
w
\end{array}\right)\left(\frac{(-1)^{p} \Gamma(j+2 v) \delta^{v} \bar{\delta}^{j}(v+j)^{p}}{\Gamma(2 v) j ! p !}\right) \\
& \times \alpha^{p+v-w} \beta^{v-w} c^{w}\left(1+x^{c}\right)^{-j-v-w} x^{\beta p-\beta w+\beta w+c w-v} d x \\
& =\sum_{j=0}^{\infty} \sum_{p=0}^{\infty} \sum_{w=0}^{v}\left(\begin{array}{c}
v \\
w
\end{array}\right)\left(\frac{(-1)^{p} \Gamma(j+2 v) \delta^{v} \bar{\delta}^{j}(v+j)^{p} \alpha^{p+v-w} \beta^{v-w} c^{w-1}}{\Gamma(2 v) j ! p !}\right) \\
& \times \int_{0}^{1} x^{\beta v-\beta w+\beta p+c w-v}\left(1+x^{c}\right)^{-j-v-w} d x \\
& =\sum_{j=0}^{\infty} \sum_{p=0}^{\infty} \sum_{w=0}^{v}\left(\begin{array}{c}
v \\
w
\end{array}\right)\left(\frac{(-1)^{p} \Gamma(j+2 v) \delta^{v} \bar{\delta}^{j}(v+j)^{p} \alpha^{p+v-w} \beta^{v-w} c^{w-1}}{\Gamma(2 v) j ! p !}\right) \\
& \times \int_{0}^{1} y^{\frac{1}{c}(\beta w-\beta v-\beta p+v+j c+v c-c-1)}(1-y)^{\frac{1}{c}(\beta v-\beta w+\beta p+c w-v+1-c)} d y \\
& =\sum_{j=0}^{\infty} \sum_{p=0}^{\infty} \sum_{w=0}^{v}\left(\begin{array}{c}
v \\
w
\end{array}\right)\left(\frac{(-1)^{p} \Gamma(j+2 v) \delta^{v} \bar{\delta}^{j}(v+j)^{p} \alpha^{p+v-w} \beta^{v-w} c^{w-1}}{\Gamma(2 v) j ! p !}\right) \\
& \times B\left(\frac{1}{c}(\beta w-\beta v-\beta p+v c+j c+v+1), \frac{1}{c}(\beta v-\beta w+\beta p+c w-v+1)\right) .
\end{aligned}
$$

Consequently, Rényi entropy is given by

$$
\begin{aligned}
I_{R}(v) & =\left(\frac{1}{1-v}\right) \log \left[\sum_{j=0}^{\infty} \sum_{p=0}^{\infty} \sum_{w=0}^{v}\left(\begin{array}{c}
v \\
w
\end{array}\right)\left(\frac{(-1)^{p} \Gamma(j+2 v) \delta^{v} \bar{\delta}^{j}(v+j)^{p} \alpha^{p+v-w} \beta^{v-w} c^{w-1}}{\Gamma(2 v) j ! p !}\right)\right] \\
& \times B\left(\frac{1}{c}(\beta w-\beta v-\beta p+v c+j c+v+1) ; \frac{1}{c}(\beta v-\beta w+\beta p+c w-v+1)\right)
\end{aligned}
$$

for $v \neq 1$, and $v>0$. 


\section{Maximum Likelihood Estimation}

Let $X \sim \operatorname{MOLLW}(c, \alpha, \beta, \delta)$ and $\boldsymbol{\Delta}=(c, \alpha, \beta, \delta)^{T}$ be the parameter vector. The $\log$-likelihood function $\ell=\ell(\boldsymbol{\Delta})$ based on a random sample of size $n$ is given by

$$
\begin{aligned}
\ell(\boldsymbol{\Delta}) & =n \log \delta-\sum_{i=0}^{n} \log \left(1+x_{i}^{c}\right)-\alpha \sum_{i=0}^{n} x_{i}^{\beta} \\
& +\sum_{i=0}^{n} \log \left\{\alpha \beta x_{i}^{\beta-1}+c x_{i}^{c-1}\left(1+x_{i}^{c}\right)^{-1}\right\}-2 \sum_{i=0}^{n} \log \left(1-\bar{\delta}\left(1+x_{i}^{c}\right)^{-1} e^{-\alpha x_{i}^{\beta}}\right) .
\end{aligned}
$$

The elements of the score function are given by

$$
\begin{gathered}
\frac{\partial \ell}{\partial \delta}=\frac{n}{\delta}-2 \sum_{i=0}^{n} \frac{\left(1+x_{i}^{c}\right)^{-1} e^{-\alpha x^{\beta}}}{1-\bar{\delta}\left(1+x_{i}^{c}\right)^{-1} e^{-\alpha x_{i}^{\beta}}}, \\
\frac{\partial \ell}{\partial \alpha}=-\sum_{i=0}^{n} x_{i}^{\beta}+\sum_{i=0}^{n} \frac{\beta x_{i}^{\beta-1}}{\alpha \beta x_{i}^{\beta-1}+c x_{i}^{c-1}\left(1+x_{i}^{c}\right)^{-1}} \\
-2 \bar{\delta} \sum_{i=0}^{n} \frac{x^{\beta}\left(1+x_{i}^{c}\right)^{-1} e^{-\alpha x_{i}^{\beta}}}{1-\bar{\delta}\left(1+x_{i}^{c}\right)^{-1} e^{-\alpha x_{i}^{\beta}}}, \\
\frac{\partial \ell}{\partial \beta}=-\alpha \sum_{i=0}^{n} x_{i}^{\beta} \log x_{i}+\sum_{i=0}^{n} \frac{\alpha x_{i}^{\beta-1}+\alpha \beta x_{i}^{\beta-1} \log x_{i}}{\alpha \beta x_{i}^{\beta-1}+c x_{i}^{c-1}\left(1+x_{i}^{c}\right)^{-1}} \\
-2 \bar{\delta} \alpha \sum_{i=0}^{n} \frac{x_{i}^{\beta}\left(1+x_{i}^{c}\right)^{-1} e^{-\alpha x_{i}^{\beta}} \log x_{i}}{1-\bar{\delta}\left(1+x_{i}^{c}\right)^{-1} e^{-\alpha x_{i}^{\beta}},}
\end{gathered}
$$

and

$$
\begin{aligned}
\frac{\partial \ell}{\partial c} & =-\sum_{i=0}^{n} \frac{x_{i}^{c} \log x_{i}}{\left(1+x_{i}^{c}\right)}-2 \alpha \sum_{i=0}^{n} \frac{\bar{\delta}\left(1+x_{i}^{c}\right)^{-2} e^{-\alpha x_{i}^{\beta}} x_{i}^{c} \log x_{i}}{1-\bar{\delta}\left(1+x_{i}^{c}\right)^{-1} e^{-\alpha x_{i}^{\beta} \log x_{i}}} \\
& +\frac{x_{i}^{c-1}\left(1+x_{i}^{c}\right)^{-1}-c x_{i}^{2 c-1}\left(1+x_{i}^{c}\right)^{-2} \log x_{i}+c x_{i}^{c-1}\left(1+x_{i}^{c}\right)^{-1} \log x_{i}}{\alpha \beta x_{i}^{\beta-1}+c x_{i}^{c-1}\left(1+x_{i}^{c}\right)^{-1}} .
\end{aligned}
$$

Note that the expectations in the Fisher Information Matrix (FIM) can be obtained numerically. Let $\hat{\Delta}=(\hat{c}, \hat{\alpha}, \hat{\beta}, \hat{\delta})$ be the maximum likelihood estimate of $\boldsymbol{\Delta}=(c, \alpha, \beta, \delta)$. Under the usual regularity conditions and that the parameters are in the interior of the parameter space, but not on the boundary, we have: $\sqrt{n}(\hat{\boldsymbol{\Delta}}-\boldsymbol{\Delta}) \stackrel{d}{\longrightarrow} N_{4}\left(\underline{0}, I^{-1}(\boldsymbol{\Delta})\right)$, where $I(\boldsymbol{\Delta})$ is the expected 
Fisher information matrix. The asymptotic behavior is still valid if $I(\boldsymbol{\Delta})$ is replaced by the observed information matrix evaluated at $\hat{\boldsymbol{\Delta}}$, that is $J(\hat{\boldsymbol{\Delta}})$. The multivariate normal distribution $N_{4}\left(\underline{0}, J(\hat{\boldsymbol{\Delta}})^{-1}\right)$, where the mean vector $\underline{\mathbf{0}}=(0,0,0,0)^{T}$, can be used to construct confidence intervals and confidence regions for the individual model parameters and for the survival and hazard rate functions. That is, the approximate $100(1-\eta) \%$ two-sided confidence intervals for $c, k, \alpha, \beta$ and $\delta$ are given by:

$$
\begin{array}{cc}
\widehat{c} \pm Z_{\frac{\eta}{2}} \sqrt{\mathbf{I}_{c c}^{-1}(\widehat{\Delta})}, & \widehat{\alpha} \pm Z_{\frac{\eta}{2}} \sqrt{\mathbf{I}_{\alpha \alpha}^{-1}(\widehat{\Delta})}, \quad \widehat{\beta} \pm Z_{\frac{\eta}{2}} \sqrt{\mathbf{I}_{\beta \beta}^{-1}(\widehat{\Delta})}, \\
\text { and } \widehat{\delta} \pm Z_{\frac{\eta}{2}} \sqrt{\mathbf{I}_{\delta \delta}^{-1}(\widehat{\Delta})},
\end{array}
$$

respectively, where $\mathbf{I}_{c c}^{-1}(\widehat{\Delta}), \mathbf{I}_{\alpha \alpha}^{-1}(\widehat{\Delta}), \mathbf{I}_{\beta \beta}^{-1}(\widehat{\Delta})$, and $\mathbf{I}_{\delta \delta}^{-1}(\widehat{\Delta})$, are the diagonal elements of $\mathbf{I}_{n}^{-1}(\hat{\boldsymbol{\Delta}})=(n \mathbf{I}(\hat{\boldsymbol{\Delta}}))^{-1}$, and $Z_{\frac{\eta}{2}}$ is the upper $\frac{\eta}{2}$ th percentile of a standard normal distribution.

\section{Simulation Study}

In this section, we study the performance and accuracy of maximum likelihood estimates of the MOLLW model parameters by conducting various simulations for different sample sizes and different parameter values. Equation 6.7 is used to generate random data from the MOELLW distribution. The simulation study is repeated for $N=$ times each with sample size $n=$ $25,50,75,100,200,400,800$ and parameter values $I: c=0.3, \alpha=1.3, \beta=$ $1.8, \delta=1.4$ and $I I: c=8.5, \alpha=0.3, \beta=0.3, \delta=0.4$. Four quantities are computed in this simulation study.

(a) Average bias of the MLE $\hat{\vartheta}$ of the parameter $\vartheta=c, \alpha, \beta, \delta$ :

$$
\frac{1}{N} \sum_{i=1}^{N}(\hat{\vartheta}-\vartheta)
$$

(b) Root mean squared error (RMSE) of the MLE $\hat{\vartheta}$ of the parameter $\vartheta=$ $c, \alpha, \beta, \delta$ :

$$
\sqrt{\frac{1}{N} \sum_{i=1}^{N}(\hat{\vartheta}-\vartheta)^{2}} .
$$

(c) Coverage probability $(\mathrm{CP})$ of $95 \%$ confidence intervals of the parameter $\vartheta=c, \alpha, \beta, \delta$, i.e., the percentage of intervals that contain the true value of parameter $\vartheta$. 
(d) Average width (AW) of $95 \%$ confidence intervals of the parameter $\vartheta=$ $c, \alpha, \beta, \delta$.

Table 8.1 presents the Average Bias, RMSE, CP and AW values of the parameters $c, \alpha, \delta$ and $\beta$ for different sample sizes. From the results, we can verify that as the sample size $n$ increases, the RMSEs decay toward zero. We also observe that for all the parametric values, the biases decrease as the sample size $n$ increases. Also, the table shows that the coverage probabilities of the confidence intervals are quite close to the nominal level of $95 \%$ and that the average confidence widths decrease as the sample size increases. Consequently, the MLE's and their asymptotic results can be used for estimating and constructing confidence intervals even for reasonably small sample sizes.

Table 8.1: Monte Carlo Simulation Results: Average Bias, RMSE, CP and AW

\begin{tabular}{|c|c|c|c|c|c|c|c|c|c|}
\hline \multirow[b]{2}{*}{ Parameter } & \multirow[b]{2}{*}{$n$} & \multicolumn{4}{|c|}{ I } & \multicolumn{4}{|c|}{ II } \\
\hline & & Average Bias & RMSE & $\mathrm{CP}$ & AW & Average Bias & RMSE & $\mathrm{CP}$ & $\mathrm{AW}$ \\
\hline \multirow[t]{7}{*}{ c } & 25 & 0.0650 & 0.1950 & 0.9230 & 0.5446 & 1.2155 & 3.2977 & 0.9360 & 9.4175 \\
\hline & 50 & 0.0275 & 0.1045 & 0.9380 & 0.3477 & 0.5025 & 1.7997 & 0.9480 & 6.0252 \\
\hline & 75 & 0.0196 & 0.0766 & 0.9460 & 0.2810 & 0.3313 & 1.3144 & 0.9470 & 4.7807 \\
\hline & 100 & 0.0159 & 0.0644 & 0.9500 & 0.2393 & 0.2378 & 1.0648 & 0.9570 & 4.0741 \\
\hline & 200 & 0.0104 & 0.0435 & 0.9540 & 0.1648 & 0.1164 & 0.7378 & 0.9430 & 2.8258 \\
\hline & 400 & 0.0067 & 0.0298 & 0.9430 & 0.1157 & 0.0455 & 0.5126 & 0.9520 & 1.9781 \\
\hline & 800 & 0.0062 & 0.0209 & 0.9550 & 0.0814 & 0.0161 & 0.3613 & 0.9470 & 1.3949 \\
\hline \multirow[t]{7}{*}{$\alpha$} & 25 & 0.0480 & 0.7388 & 0.8800 & 2.4684 & 0.1009 & 0.4749 & 0.8590 & 0.9655 \\
\hline & 50 & 0.0185 & 0.4615 & 0.9230 & 1.7089 & 0.0308 & 0.1965 & 0.9190 & 0.4769 \\
\hline & 75 & 0.0091 & 0.3771 & 0.9320 & 1.3983 & 0.0140 & 0.0877 & 0.9210 & 0.3338 \\
\hline & 100 & -0.0009 & 0.3176 & 0.9290 & 1.1968 & 0.0107 & 0.0774 & 0.9230 & 0.2810 \\
\hline & 200 & -0.0207 & 0.2103 & 0.9440 & 0.8289 & 0.0041 & 0.0492 & 0.9440 & 0.1877 \\
\hline & 400 & -0.0070 & 0.1510 & 0.9400 & 0.5865 & 0.0017 & 0.0329 & 0.9470 & 0.1297 \\
\hline & 800 & -0.0182 & 0.1047 & 0.9560 & 0.4128 & 0.0015 & 0.0236 & 0.9480 & 0.0914 \\
\hline \multirow[t]{7}{*}{$\beta$} & 25 & 0.8538 & 2.3429 & 0.9320 & 4.1588 & 0.0411 & 0.1411 & 0.9570 & 0.4766 \\
\hline & 50 & 0.2740 & 0.7255 & 0.9540 & 2.1730 & 0.0174 & 0.0839 & 0.9570 & 0.3044 \\
\hline & 75 & 0.1521 & 0.4895 & 0.9450 & 1.6559 & 0.0128 & 0.0668 & 0.9480 & 0.2420 \\
\hline & 100 & 0.1122 & 0.3966 & 0.9550 & 1.3962 & 0.0117 & 0.0546 & 0.9670 & 0.2091 \\
\hline & 200 & 0.0638 & 0.2538 & 0.9570 & 0.9481 & 0.0071 & 0.0384 & 0.9440 & 0.1453 \\
\hline & 400 & 0.0390 & 0.1763 & 0.9370 & 0.6534 & 0.0032 & 0.0257 & 0.9510 & 0.1011 \\
\hline & 800 & 0.0269 & 0.1211 & 0.9480 & 0.4592 & 0.0032 & 0.0182 & 0.9550 & 0.0715 \\
\hline \multirow[t]{7}{*}{$\delta$} & 25 & 0.3599 & 3.3465 & 0.8260 & 6.9270 & 0.6220 & 6.2480 & 0.8610 & 9.5925 \\
\hline & 50 & 0.09897 & 0.8351 & 0.8810 & 2.8645 & 0.0992 & 1.3014 & 0.9230 & 1.1467 \\
\hline & 75 & 0.0663 & 0.6073 & 0.8970 & 2.2168 & 0.0225 & 0.1540 & 0.9280 & 0.5844 \\
\hline & 100 & 0.0408 & 0.5245 & 0.9050 & 1.8401 & 0.0184 & 0.1382 & 0.9420 & 0.4948 \\
\hline & 200 & -0.0088 & 0.3111 & 0.9170 & 1.2004 & 0.0076 & 0.0845 & 0.9420 & 0.3299 \\
\hline & 400 & -0.0027 & 0.2196 & 0.9360 & 0.8447 & 0.0029 & 0.0576 & 0.9600 & 0.2277 \\
\hline & 800 & -0.0179 & 0.1492 & 0.9360 & 0.5858 & 0.0015 & 0.0428 & 0.9390 & 0.1606 \\
\hline
\end{tabular}

\section{Applications}

In this section, we present examples to illustrate the flexibility of the MOLLW distribution and its sub-models for data modeling. We fit the density function 
of the MOLLW, Marshall-Olkin log logistic exponential (MOLLE), MarshallOlkin log logistic Rayleigh (MOLLR), Marshall-Olkin log logistic and log logistic Weibull (LLW) distributions. We also compare the MOLLW distribution to other models including the gamma-Dagum (GD) (Oluyede et al. 2016) and beta Weibull (Lee et al. 2007) distributions. The GD and BW pdfs are given by

$$
g_{G D}(x)=\frac{\lambda \beta \delta x^{-\delta-1}}{\Gamma(\alpha)}\left(1+\lambda x^{-\delta}\right)^{-\beta-1}\left(-\log \left[1-\left(1+\lambda x^{-\delta}\right)^{-\beta}\right]\right)^{\alpha-1}, \quad x>0,
$$

and

$$
g_{B W}(x)=\frac{k \lambda^{k}}{B(a, b)} x^{k-1} e^{-b(\lambda x)^{k}}\left(1-e^{-(\lambda x)^{k}}\right)^{a-1}, \quad x>0,
$$

respectively. The maximum likelihood estimates (MLEs) of the MOLLW parameters $c, \alpha, \beta, \delta$ are computed by maximizing the objective function via the subroutine NLMIXED in SAS as well (bbmle) package in R. The estimated values of the parameters (standard error in parenthesis), -2log-likelihood statistic, Akaike Information Criterion, $A I C=2 p-2 \ln (L)$, Consistent Akaike Information Criterion, $\left(A I C C=A I C+\frac{2 p(p+1)}{n-p-1}\right)$ and Bayesian Information Criterion, $B I C=p \ln (n)-2 \ln (L)$, where $L=L(\hat{\Delta})$ is the value of the likelihood function evaluated at the parameter estimates, $n$ is the number of observations, and $p$ is the number of estimated parameters are presented in Tables 9.2 and 9.4 for the MOLLW distribution and its sub-models MOLLE, MOLLR, MOLL, LLW, LLE and LW distributions and alternatives (non-nested) GD and BW distributions. The Cramer von Mises and Anderson-Darling goodness-of-fit statistics $W^{*}$ and $A^{*}$, are also presented in these tables. These statistics can be used to verify which distribution fits better to the data. In general, the smaller the values of $W^{*}$ and $A^{*}$, the better the fit. The AdequacyModel package was used to evaluate the statistics stated above.

We can use the likelihood ratio (LR) test to compare the fit of the MOLLW distribution with its sub-models for a given data set. For example, to test $\beta=1$, the $\mathrm{LR}$ statistic is $\omega=2[\ln (L(\hat{c}, \hat{\alpha}, \hat{\beta}, \hat{\delta}))-\ln (L(\tilde{c}, \tilde{\alpha}, 1, \tilde{\delta}))]$, where $\hat{c}$, $\hat{\alpha}, \hat{\beta}$ and $\hat{\delta}$ are the unrestricted estimates, and $\tilde{c}, \tilde{\alpha}$ and $\tilde{\delta}$ are the restricted estimates. The LR test rejects the null hypothesis if $\omega>\chi_{\epsilon}^{2}$, where $\chi_{\epsilon}^{2}$ denote the upper $100 \epsilon \%$ point of the $\chi^{2}$ distribution with 1 degrees of freedom.

\subsection{Glass Fibers Data}

Specifically we consider the following data set which consists of 63 observations of the strengths of $1.5 \mathrm{~cm}$ glass fibers, originally obtained by workers at the UK National Physical Laboratory. The data was also studied by (Smith and Naylor 1987). The data observations are given below: 
Table 9.1: Glass fibers data

\begin{tabular}{llllll}
\hline 0.55 & 0.93 & 1.25 & 1.36 & 1.49 & 1.52 \\
1.58 & 1.61 & 1.64 & 1.68 & 1.73 & 1.81 \\
2.00 & 0.74 & 1.04 & 1.27 & 1.39 & 1.49 \\
1.53 & 1.59 & 1.61 & 1.66 & 1.68 & 1.76 \\
1.82 & 2.01 & 0.77 & 1.11 & 1.28 & 1.42 \\
1.50 & 1.54 & 1.60 & 1.62 & 1.66 & 1.69 \\
1.76 & 1.84 & 2.24 & 0.81 & 1.13 & 1.29 \\
1.48 & 1.50 & 1.55 & 1.61 & 1.62 & 1.66 \\
1.70 & 1.77 & 1.84 & 0.84 & 1.24 & 1.30 \\
1.48 & 1.51 & 1.55 & 1.61 & 1.63 & 1.67 \\
1.70 & 1.78 & 1.89 & & & \\
\hline
\end{tabular}

Estimates of the parameters of MOLLW distribution and its related submodels (standard error in parentheses), $-2 \ln (L)$, AIC, AICC, BIC, $W^{*}$ and $A^{*}$ are given in Table 9.2. Initial values for the MOLLW model in $\mathrm{R}$ code are $(c=0.06, \alpha=0.15, \beta=0.15, \delta=100.4)$.

Table 9.2: Estimates of Models for Glass fibers Data

\begin{tabular}{|c|c|c|c|c|c|c|c|c|c|c|}
\hline \multirow[b]{2}{*}{ Model } & \multicolumn{4}{|c|}{ Estimates } & \multicolumn{4}{|c|}{ Statistics } & \multirow[b]{2}{*}{$W^{*}$} & \multirow[b]{2}{*}{$A^{*}$} \\
\hline & $\hat{c}$ & $\hat{\alpha}$ & $\hat{\beta}$ & $\hat{\delta}$ & $-2 \log L$ & $A I C$ & $A I C C$ & $B I C$ & & \\
\hline MOLLW & $\begin{array}{c}2.3671 \\
(1.8197)\end{array}$ & $\begin{array}{c}0.3779 \\
(0.6353)\end{array}$ & $\begin{array}{c}3.7953 \\
(1.9556)\end{array}$ & $\begin{array}{c}29.1187 \\
(37.8767)\end{array}$ & 23.7877 & 31.7877 & 32.4773 & 40.3603 & 0.0862 & 0.4963 \\
\hline MOLLE & $\begin{array}{c}5.9702 \\
(1.5263)\end{array}$ & $\begin{array}{c}1.7415 \\
(0.9350)\end{array}$ & $\begin{array}{l}1 \\
-\end{array}$ & $\begin{array}{c}196.7129 \\
(197.3287)\end{array}$ & 32.0457 & 42.0457 & 42.4525 & 48.4751 & 0.2956 & 1.6423 \\
\hline MOLLR & $\begin{array}{c}2.9412 \\
(2.1648)\end{array}$ & $\begin{array}{c}1.2508 \\
(0.4351)\end{array}$ & $\begin{array}{l}2 \\
-\end{array}$ & $\begin{array}{c}81.8760 \\
(39.8069)\end{array}$ & 29 . & 35.1556 & 35.5624 & 41.5853 & 0.2049 & 1.1245 \\
\hline MOLL & $\begin{array}{c}7.9224 \\
(0.8725)\end{array}$ & - & - & $\begin{array}{c}28.4663 \\
(12.7027)\end{array}$ & 45.5800 & 49.579 & 45.78 & 53.8662 & 0.4971 & 2.7498 \\
\hline LLW & $\begin{array}{c}1.2716) \\
(0.3961)\end{array}$ & $\begin{array}{r}0.00885) \\
(0.0053)\end{array}$ & $\begin{array}{c}8.4472 \\
(0.8923)\end{array}$ & - & 20 & 96.0120 & 96.4188 & 103.0425 & 0.4970 & 2.7498 \\
\hline GD & $\begin{array}{c}\hat{\lambda} \\
68.4591 \\
(38.2861) \\
\hat{\lambda}\end{array}$ & $\begin{array}{c}\hat{\beta} \\
0.08732 \\
(0.1939) \\
\hat{k}\end{array}$ & $\begin{array}{c}\hat{\delta} \\
11.2921 \\
(3.1002) \\
\hat{a}\end{array}$ & $\begin{array}{c}\hat{\alpha} \\
3.4731 \\
(3.1763) \\
\hat{b}\end{array}$ & 36.9692 & 44.9692 & 45.6592 & 53.5417 & 0.3510 & 1.9284 \\
\hline BW & $\begin{array}{c}0.3046 \\
(0.1019)\end{array}$ & $\begin{array}{l}4.4451 \\
0.8567\end{array}$ & $\begin{array}{c}1.1987 \\
(0.35094)\end{array}$ & $\begin{array}{l}28.8812 \\
41.2092\end{array}$ & 33 & 41.4283 & 42.1179 & 50.0082 & 0.2878 & 1.5770 \\
\hline
\end{tabular}

The asymptotic covariance matrix of the MLE's for the MOLLW model parameters which is the observed Fisher information matrix $\mathbf{I}_{n}^{-1}(\widehat{\Delta})$ is given by:

$$
\left(\begin{array}{cccc}
2.3661466 & -0.3889304 & 1.4554194 & -12.846596 \\
-0.3889304 & 0.1374642 & -0.5817194 & 6.667768 \\
1.4554194 & -0.5817194 & 2.5332639 & -28.197423 \\
-12.8465957 & 6.6677685 & -28.1974230 & 396.969514
\end{array}\right)
$$


and the $95 \%$ confidence intervals for the model parameters are given by $c \in$ $(2.3671 \pm 1.96 \times 1.8197), \alpha \in(0.3779 \pm 1.96 \times 0.6353), \beta \in(3.7953 \pm 1.96 \times 1.9556)$ and $\delta \in(29.1187 \pm 1.96 \times 37.8767)$, respectively.

The LR test statistic of the hypothesis $H_{0}$ : MOLLE against $H_{a}$ : MOLLW, $H_{0}$ : MOLLR against $H_{a}$ : MOLLW, and $H_{0}$ : MOLL against $H_{a}$ : MOLLW and LLW against $H_{a}$ : MOLLW, are 8.258 (p-value $\left.=0.0045\right), 5.3679(\mathrm{p}$-value $=0.02051), 21.7923\left(\mathrm{p}\right.$-value $\left.=1.96 \times 10^{-5}\right)$ and $66.2243(\mathrm{p}$-value $=0.00001)$. We can conclude that there are significant differences between MOLLW and its sub-models: MOLLE, MOLLR, MOLL, and LLW distributions at the $5 \%$ level of significance. The values of the statistics: AIC, AICC and BIC also shows that the MOLLW distribution is a better fit than the non-nested GD and BW distributions for the glass fibers data. There is also clear evidence based on the goodness-of-fit statistics $W^{*}$ and $A^{*}$ that the MOLLW distribution is by far the better fit for the glass fibers data. Plot of the fitted densities, and the histogram of the data are given in Figure 9.1.

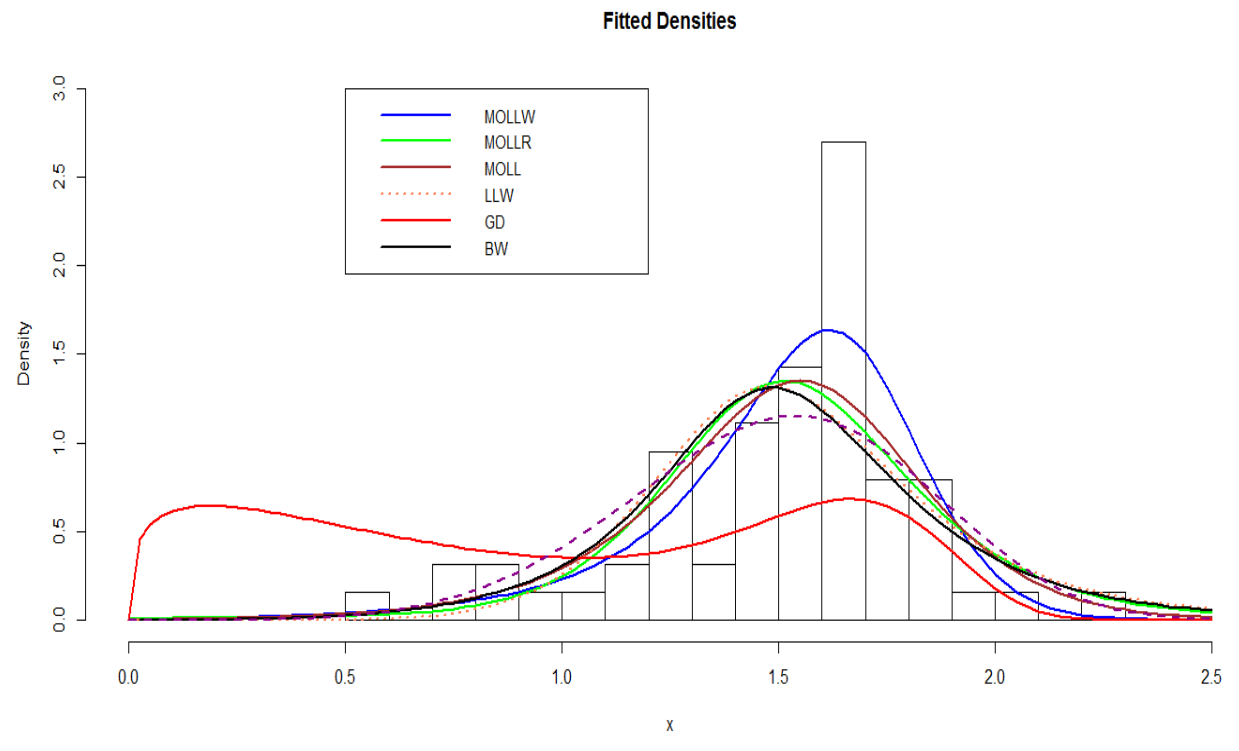

Figure 9.1: Fitted densities for glass fiber data

\subsection{Breaking Stress of Carbon Fibres (in Gba) Data}

This data set consists of 66 uncensored data on breaking stress of carbon fibres (in Gba) (Nicholas and Padgett 2006). The data values are given Table 9.3.

Estimates of the parameters of MOLLW distribution and its related submodels (standard error in parentheses), $-2 \ln (L)$, AIC, AICC, and BIC are given in Table 9.2. Initial values for the MOLLW model in $\mathrm{R}$ code are $(c=$ $5.326, \alpha=0.15, \beta=1.56, \delta=1.4$ ). 
Table 9.3: Carbon fibers data

\begin{tabular}{llllll}
\hline 0.39 & 0.85 & 1.08 & 1.25 & 1.47 & 1.57 \\
1.61 & 1.61 & 1.69 & 1.80 & 1.84 & 1.87 \\
1.89 & 2.03 & 2.03 & 2.05 & 2.12 & 2.35 \\
2.41 & 2.43 & 2.48 & 2.50 & 2.53 & 2.55 \\
2.55 & 2.56 & 2.59 & 2.67 & 2.73 & 2.74 \\
2.79 & 2.81 & 2.82 & 2.85 & 2.87 & 2.88 \\
2.93 & 2.95 & 2.96 & 2.97 & 3.09 & 3.11 \\
3.11 & 3.15 & 3.15 & 3.19 & 3.22 & 3.22 \\
3.27 & 3.28 & 3.31 & 3.31 & 3.33 & 3.39 \\
3.39 & 3.56 & 3.60 & 3.65 & 3.68 & 3.70 \\
3.75 & 4.20 & 4.38 & 4.42 & 4.70 & 4.90 \\
\hline
\end{tabular}

Table 9.4: Estimates of Models for Carbon fibers Data

\begin{tabular}{|c|c|c|c|c|c|c|c|c|c|c|}
\hline \multirow[b]{2}{*}{ Model } & \multicolumn{4}{|c|}{ Estimates } & \multicolumn{4}{|c|}{ Statistics } & \multirow[b]{2}{*}{$W^{*}$} & \multirow[b]{2}{*}{$A^{*}$} \\
\hline & $\hat{c}$ & $\hat{\alpha}$ & $\hat{\beta}$ & $\hat{\delta}$ & $-2 \log L$ & $A I C$ & $A I C C$ & $B I C$ & & \\
\hline MOLLW & $\begin{array}{c}1.6752 \\
(1.5051)\end{array}$ & $\begin{array}{c}0.2786 \\
(0.6144)\end{array}$ & $\begin{array}{c}1.9393 \\
(1.1526)\end{array}$ & $\begin{array}{c}50.1701 \\
(57.8832)\end{array}$ & 169.4 & 177.4 & 178.1 & 186.2 & 0.0404 & 0.2643 \\
\hline MOLL & $\begin{array}{c}4.8958 \\
(0.5137)\end{array}$ & - & - & $\begin{array}{c}131.97 \\
(75.1761)\end{array}$ & 183.3 & 187.3 & 187.5 & 191.7 & 0.0961 & 0.8804 \\
\hline LLW & $\begin{array}{c}0.3318 \\
(0.1703)\end{array}$ & $\begin{array}{c}0.00568 \\
(0.00442)\end{array}$ & $\begin{array}{l}4.3347 \\
(0.55)\end{array}$ & - & 252.7 & 258.1 & 259.1 & 265.3 & 0.2167 & 1.8405 \\
\hline LLE & $\begin{array}{c}1.5296 \\
(0.1473) \\
\hat{\lambda}\end{array}$ & $\begin{array}{c}0.3624 \\
(0.4461) \\
\hat{\beta}\end{array}$ & $\begin{array}{l}1 \\
\hat{\delta}\end{array}$ & $\hat{\alpha}$ & 591.2 & 595.2 & 595.4 & 599.6 & 0.3492 & 1.9224 \\
\hline GD & $\begin{array}{c}5.271 \\
(2.5145) \\
\hat{\lambda}\end{array}$ & $\begin{array}{c}8.7229 \\
(1.4628) \\
\hat{k}\end{array}$ & $\begin{array}{c}2.6974 \\
(0.2773) \\
\hat{a}\end{array}$ & $\begin{array}{c}0.2653 \\
(0.004730) \\
\hat{b}\end{array}$ & 198.2 & 206.2 & 206.8 & 214.9 & 0.4380 & 2.4110 \\
\hline BW & $\begin{array}{c}0.1886 \\
(0.01883)\end{array}$ & $\begin{array}{c}4.0692 \\
(1.2754)\end{array}$ & $\begin{array}{c}0.7601 \\
(0.3697)\end{array}$ & $\begin{array}{c}6.6366 \\
(2.3168)\end{array}$ & 171.8 & 179.8 & 180.5 & 188.6 & 0.0846 & 0.5040 \\
\hline
\end{tabular}

The asymptotic covariance matrix of the MLE's for the MOLLW model parameters which is the observed Fisher information matrix $\mathbf{I}_{n}^{-1}(\widehat{\Delta})$ is given by:

$$
\left(\begin{array}{cccc}
2.2655 & -0.7684 & 1.3251 & -45.3059 \\
-0.7684 & 0.3775 & -0.7018 & 31.4482 \\
1.3251 & -0.7018 & 1.3285 & -60.1852 \\
-54.3059 & 31.4482 & -60.1852 & 3350.46
\end{array}\right)
$$

and the $95 \%$ confidence intervals for the model parameters are given by $c \in$ $(1.6752 \pm 1.96 \times 1.5051), \alpha \in(0.2786 \pm 1.96 \times 0.6144), \beta \in(1.9393 \pm 1.96 \times 1.1526)$ and $\delta \in(50.1701 \pm 1.96 \times 57.8832)$, respectively.

The LR test statistic of the hypothesis $H_{0}$ : LLW against $H_{a}$ : MOLLW, $H_{0}$ : LLE against $H_{a}$ : MOLLW, and $H_{0}$ : MOLL against $H_{a}$ : MOLLW and are $83.3(\mathrm{p}$-value $=0.00001), 421.8(\mathrm{p}$-value $=0.00001), 13.9(\mathrm{p}$-value $=0.000959)$. 
We can conclude that there are significant differences between MOLLW and the sub-models: LLW, LLE, and MOLL distributions at the $5 \%$ level of significance. The values of the statistics: AIC, AICC and BIC also shows that the MOLLW distribution is a better fit than the non-nested GD and BW distributions for the carbon fiber data. Infact, there is also clear evidence based on the Cramer von Mises and Anderson-Darling goodness-of-fit statistics $W^{*}$ and $A^{*}$ that the MOLLW distribution is by far the better fit for the carbon fiber data. Plot of the fitted densities, and the histogram of the data are given in Figure 9.2.

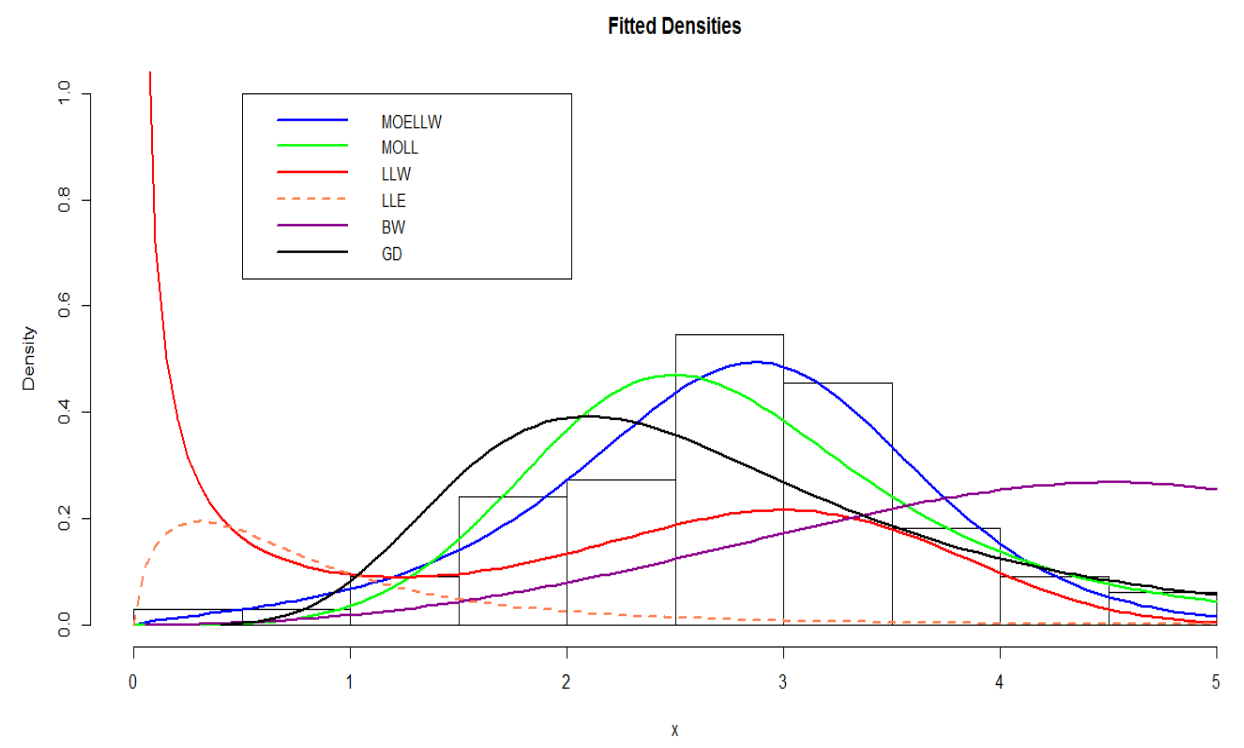

Figure 9.2: Fitted densities for carbon fiber data

\section{Concluding Remarks}

A new class of distributions called the MOLLEW distribution and its special case MOLLW are presented. This general and specific class of distributions and some of its structural properties including hazard and reverse hazard functions, quantile function, moments, conditional moments, Bonferroni and Lorenz curves, Rényi entropy, maximum likelihood estimates, asymptotic confidence intervals are presented. Applications of the specific MOLLW model to real data sets are given in order to illustrate the applicability and usefulness of the proposed distribution. MOLLW distribution has a better fit than some of its sub-models and the non-nested GD and BW distributions for the glass fibers data and carbon fibers data. 


\section{References}

[1] Barreto-Souza, W., and Bakouch., H. S. (2013). A new lifetime model with decreasing failure rate, Statistics: A Journal of Theoretical and Applied Statistics, 47, 465-476.

[2] Bebbington M., Lai C. D., and Zitikis R. (2007). A flexible Weibull extension, Reliability Engineering System Safety, 92, 719-726.

[3] Cordeiro, G. M., Ortega E. M., and Nadarajah S. (2010). The Kumaraswamy Weibull Distribution with Applications to Failure Data, Journal of Franklin Institute, 347, 1399-1429.

[4] Cordeiro, G. M., and Lemonte A. J. A. (2011). On the Marshall-Olkin extended Weibull distribution, Stat. Paper, 54, 333-353.

[5] Chakraborty, S., and Handique, L. (2017). The Generalized MarshallOlkin-Kumaraswamy-G Family of Distributions, Journal of Data Science, 15(3), 391-422.

[6] Lee C., Famoye F., and Olumolade O. (2007). Beta-Weibull Distribution: Some Properties and Applications, Journal of Modern Applied Statistical Methods, 6, 173-186.

[7] Ghitany, M. E, AL-Hussaini, E. K., and AL-Jarallah. (2005). MarshallOlkin Extended Weibull distribution and its application to Censored data, Journal of Applied Statistics, 32(10), 1025-1034.

[8] Gurvich, M. R., DiBenedetto, A. T., and Ranade, S. V. (1997). A new statistical distribution for characterizing the random strength of brittle materials, Journal of Materials Science 32, 2559-2564.

[9] Hjorth, U. (1980). A Reliability Distribution With Increasing, Decreasing, Constant and Bathtub Failure Rates, Technometrics, 22, 99-107.

[10] Kumar, D. (2016). Ratio and Inverse Moments of Marshall-Olkin Extended Burr Type III Distribution Based on Lower Generalized Order Statistics, Journal of Data Science, 14(1), 53-66.

[11] Lazhar, B. (2017). Marshall-Olkin Extended Generalized Gompertz Distribution, Journal of Data Sciences, 15(2), 239-266.

[12] Marshall A. W., and Olkin I. (1997). A New Method for Adding a Parameter to a Family of Distributions with Application to the Exponential and Weibull Families, Biometrika 84: 641-652. 
[13] Nichols, M. D., and Padgett, W. J. (2006). A Bootstrap control chart for Weibull percentiles, Quality and Reliability Engineering International, 22, 141-151.

[14] Oluyede, B. O., Huang, S., and Pararai, M. (2014). A New Class of Generalized Dagum Distribution with Applications to Income and Lifetime Data, Journal of Statistical and Econometric Methods, 3(2), 125-151.

[15] Oluyede, B. O., Foya, S., Warahena-Liyanage, G., and Huang, S. (2016). The Log-logistic Weibull Distribution with Applications to Lifetime Data, Autrian Journal of Statistics, 45, 43-69.

[16] Rényi, A. (1960). On Measures of Entropy and Information, Proceedings of the Fourth Berkeley Symposium on Mathematical Statistics and Probability, 1, 547 - 561 .

[17] Santos-Neo, M., Bourguignon, M., Zea, L. M., and Nascimento, A. D. C. (2014). The Marshall-Olkin Extended Weibull Family of Distributions, Journal of Statistical Distributions and Applications, 1-9.

[18] Silva, G. O., Ortega, E. M., and Cordeiro, G. M. (2010). The Beta Modified Weibull Distribution, Lifetime Data Analysis, 16, 409-430.

[19] Smith, R. L., and Naylor, J. C. (1987). A comparison of maximum likelihood and Bayesian estimators for the three-parameter Weibull distribution. Applied Statistics, 36, 358-369.

[20] Xie, M., Tang Y., Goh T. N. (2009). A modified Weibull extension with bathtub-shaped failure rate function, Reliability and Engineering System Safety, 76,279-285.

[21] Zhang, T., and Xie M. (2007). Failure data analysis with extended Weibull distribution, Commun. Stat. Simul., 36 579-592. 


\section{A R Code: Define Functions}

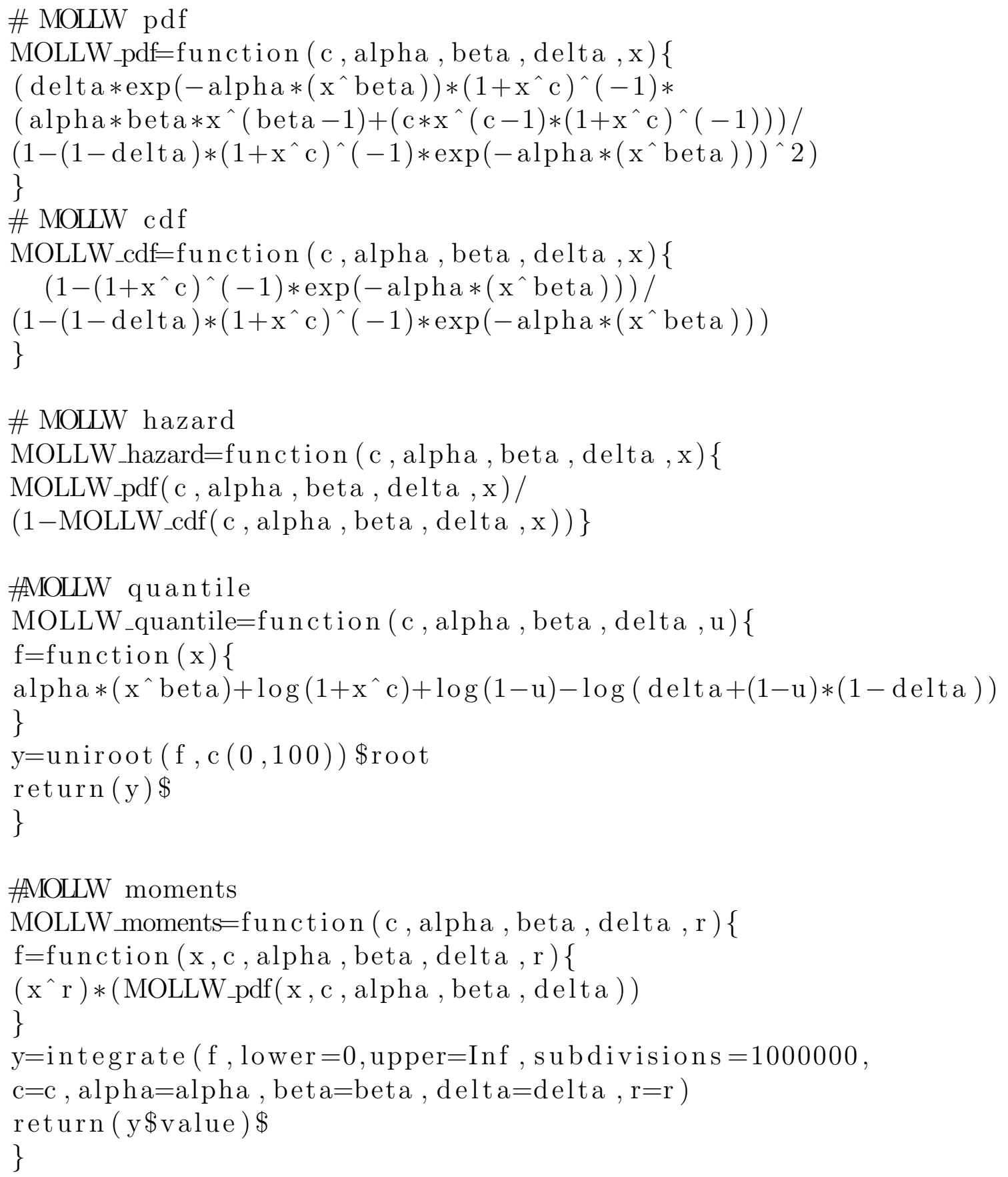




\section{Lornah Lepetu}

Department of Mathematics and Statistical Sciences

Botswana International University of Science and Technology, Palapye, BW

Broderick O. Oluyede

Department of Mathematical Sciences

Georgia Southern University, GA 30460, USA

boluyede@georgiasouthern.edu

Boikanyo Makubate

Department of Mathematics and Statistical Sciences

Botswana International University of Science and Technology, Palapye, BW

Susan Foya

First National Bank

Gaborone, BW

Precious Mdlongwa

Department of Mathematics and Statistical Sciences

Botswana International University of Science and Technology, Palapye, BW 
